\title{
WLS-ENO: Weighted-Least-Squares Based Essentially Non-Oscillatory Schemes for Finite Volume Methods on Unstructured Meshes
}

\author{
Hongxu Liu, Xiangmin Jiao* \\ Department of Applied Mathematics and Statistics, Stony Brook University \\ Stony Brook, NY 11794, USA
}

\begin{abstract}
ENO (Essentially Non-Oscillatory) and WENO (Weighted Essentially Non-Oscillatory) schemes are widely used high-order schemes for solving partial differential equations (PDEs), especially hyperbolic conservation laws with piecewise smooth solutions. For structured meshes, these techniques can achieve high order accuracy for smooth functions while being non-oscillatory near discontinuities. For unstructured meshes, which are needed for complex geometries, similar schemes are required but they are much more challenging. We propose a new family of non-oscillatory schemes, called $W L S$-ENO, in the context of solving hyperbolic conservation laws using finite-volume methods over unstructured meshes. WLS-ENO is derived based on Taylor series expansion and solved using a weighted least squares formulation. Unlike other non-oscillatory schemes, the WLS-ENO does not require constructing sub-stencils, and hence it provides a more flexible framework and is less sensitive to mesh quality. We present rigorous analysis of the accuracy and stability of WLS-ENO, and present numerical results in 1-D, 2-D, and 3-D for a number of benchmark problems, and also report some comparisons against WENO.
\end{abstract}

Keywords: essentially non-oscillatory scheme; weighted least squares; finite volume method; hyperbolic conservation law; unstructured mesh

\section{Introduction}

Many physical phenomena, such as waves, heat conduction, electrodynamics, elasticity, etc., can be modeled by partial differential equations. With the development of computer technology, many numerical methods have been designed to solve these kinds of problems over the past decades. Among these there are finite difference methods and their generalizations, finite volume methods, and finite element methods.

In this paper, we consider the problem of reconstructing a piecewise smooth function, in the context of finite volume methods for hyperbolic conservation laws. Given a geometric domain $\Omega \subseteq \mathbb{R}^{d}$, suppose $u$ is a time-dependent piecewise smooth function over $\Omega$, such as a density function. For any connected region $\tau$, the $d$-dimensional conservation law can be written in the form

$$
\int_{\tau} \frac{\partial u(\boldsymbol{x}, t)}{\partial t} d \boldsymbol{x}=-\int_{\partial \tau} \boldsymbol{F}(u) \cdot d \boldsymbol{a},
$$

where $\partial \tau$ is the boundary of $\tau$, and $\boldsymbol{F}$ is a function of $u$, corresponding to the flux.

A finite volume method solves the problem by decomposing the domain $\Omega$ into cells $\left\{\tau_{i} \mid i=1, \ldots, N\right\}$. Let $\left|\tau_{i}\right|$ denote the volume of $\tau_{i}$ and $\bar{u}_{i}(t)=\frac{1}{\left|\tau_{i}\right|} \int_{\tau_{i}} u(\boldsymbol{x}, t) d \boldsymbol{x}$, the average of $u$ over $\tau_{i}$. We obtain an equation

$$
\frac{d \bar{u}_{i}(t)}{\partial t}=-\left|\tau_{i}\right| \int_{\partial \tau_{i}} \boldsymbol{F}(u) \cdot d \boldsymbol{a},
$$

for each $\tau_{i}$. The boundary integral requires using numerical quadrature for the flux. The integration of the flux requires reconstructing $u$ from the cell averages $\bar{u}(t)$ in an accurate and stable fashion, and then

* Corresponding author.

Email address: xiangmin.jiao@stonybrook.edu (Xiangmin Jiao) 
evaluating the reconstruction at the quadrature points along the cell boundaries. For stability, $\boldsymbol{F} \cdot \boldsymbol{n}$ is typically replaced by a numerical flux, such as the Lax-Friedrichs flux,

$$
\boldsymbol{F} \cdot \boldsymbol{n}=\frac{1}{2}\left[\left(\boldsymbol{F}\left(u^{-}\right)+\boldsymbol{F}\left(u^{+}\right)\right) \cdot \boldsymbol{n}-\alpha\left(u^{+}-u^{-}\right)\right],
$$

where $u^{-}$and $u^{+}$are the values of $u$ inside and outside the cell $\tau_{i}$. The parameter $\alpha$ is a constant, and it should be an upper bound of the eigenvalues of the Jacobian of $u$ in the normal direction.

In this context, we formulate the mathematical problem addressed in this paper as follows: Given the cell averages $\bar{u}_{i}$ of a piecewise smooth function $u(\boldsymbol{x})$ for cell $\tau_{1}, \tau_{2}, \ldots, \tau_{N}$, let $h_{i}$ be some length measure of cell $\tau_{i}$. Find a polynomial approximation $\widetilde{u}_{i}(\boldsymbol{x})$ of degree at most $p-1$ over $\tau_{i}$, such that

$$
\left\|\widetilde{u}_{i}(\boldsymbol{x})-u_{i}(\boldsymbol{x})\right\|=\mathcal{O}\left(h_{i}^{p}\right), \quad \boldsymbol{x} \in \tau_{i} .
$$

In other words, $\widetilde{u}_{i}(\boldsymbol{x})$ is a $p$ th order accurate approximation to $u(\boldsymbol{x})$ inside $\tau_{i}$. In the context of hyperbolic conservation laws, $u(\boldsymbol{x})$ in (4) is equal to $u(\boldsymbol{x}, t)$ in (1) at a given $t$. For the facet between two cells, these reconstructions give us two values $u^{-}$and $u^{+}$, which can then be substituted into (3) to calculate the numerical flux. These reconstructions must be accurate, and also must lead to stable discretizations of the hyperbolic conservation laws when coupled with some appropriate time integration schemes, such as TVD Runge-Kutta schemes [1].

This reconstruction problem is decidedly challenging, because hyperbolic conservation laws can produce non-smooth solutions. An approximation scheme for smooth functions may lead to oscillations that do not diminish as the mesh is refined, analogous to the Gibbs phenomena. Such oscillations would undermine the convergence of the solutions. The ENO (Essentially Non-Oscillatory) and WENO (Weighted Essentially Non-Oscillatory) schemes $[2,3,4]$ have been successful in solving this problem. In a nutshell, the WENO schemes use a convex combination of polynomials constructed over some neighboring cells, with higher weights for cells with smoother solutions and lower weights for cells near discontinuities. As a result, these methods can achieve high-order accuracy at smooth regions while being non-oscillatory near discontinuities. These reconstructions can be integrated into both finite volume and finite difference methods. With years of development, finite volume WENO schemes have been applied to both structured and unstructured meshes and higher dimensions $[5,6,7,8,9,10]$. Various attempts have been applied to improve the weights for WENO reconstruction $[11,12,13,14]$. Also, they have used WENO schemes in many applications, such as shock vortex interaction [15], incompressible flow problems [16], Hamilton-Jacobi equations [17], shallow water equations [18], etc.

Along the path of applying WENO schemes on unstructured meshes, tremendous effort has been made to improve the robustness of the schemes. Early attempts [5] work well for most unstructured meshes, but some point distributions may lead to negative weights and in turn make the schemes unstable. An extension was proposed in [7] to mitigate the issue, but it still had limited success over complicated geometries due to inevitably large condition numbers of their local linear systems. More recently, several different partition techniques were proposed to improve stability, such as [19], which uses a hybrid of two different reconstruction strategies to achieve better results. The technique was adopted in [20, 21, 22] for further development.

In this paper, we propose a new family of reconstruction methods over unstructured meshes. We refer to the schemes as WLS-ENO, or Weighted-Least-Squares based Essentially Non-Oscillatory schemes. Unlike the WENO scheme, our approach uses a generalized finite difference (GFD) formulation based on weighted least squares, rather than a weighted averaging of traditional finite differences. The GFD method is derived rigorously from Taylor series, and hence can deliver the same order of accuracy as traditional finite differences. In WLS-ENO, the convexity requirement is satisfied automatically, since the weights are specified a priori. These properties enable a more systematic way to construct non-oscillatory schemes. We will present the detailed derivation of the schemes and their robust numerical solution techniques. We also show that the schemes are often more accurate than WENO schemes near discontinuities and enable more stable PDE solvers when used in conjunction with total variation-diminishing time-integration schemes such as TVD Runge-Kutta. We report theoretical analysis in 1-D as well as experimental results in 1-D, 2-D, and 3-D.

The remainder of this paper is organized as follows. Section 2 reviews the ENO and WENO schemes, as well as some related background knowledge. Section 3 presents the derivation and numerical methods of the WLS-ENO schemes. Section 4 analyzes the accuracy and stability of the WLS-ENO schemes, and 
compares them against WENO and its previous generalization to unstructured meshes. Section 5 presents some numerical results and comparisons against some other methods. Finally, Section 6 concludes the paper with some discussions on future research directions.

\section{Background and Related Work}

In this section, we present some background information, including WENO schemes and some related numerical methods for hyperbolic conservation laws. These will motivate the derivation of WLS-ENO.

Traditionally, two approaches have been used to reduce oscillations near discontinuities. One approach was to add artificial viscosity [23, 24]. Specifically, one could design the viscosity to be larger near discontinuity to suppress oscillations and to be smaller elsewhere to maintain accuracy. However, the parameter controlling the artificial viscosity is very problem dependent. Another approach was to apply limiters $[23,24]$, but such schemes degenerate to first order near discontinuities. Other successful methods include ENO scheme [25] and its closely related WENO schemes [2]. In a nutshell, the ENO is a WENO scheme with only zeros and ones as the weights. In the following, we review some of the ENO and WENO schemes that are most closely related to our proposed approach.

\subsection{WENO Reconstructions in 1-D}

In the context of finite volume methods, the basic idea of WENO is to first construct several stencils for each cell and local polynomials over these stencils, so that the cell averages of these polynomials are the same as the given values. Then, a WENO scheme uses a convex combination of these polynomials to obtain a reconstruction of the function, where the weights for each stencil are controlled by a smoothness indicator. We briefly describe the WENO scheme on a uniform 1-D grid below, and refer readers to [2] for more detail.

Given a 1-D domain $[a, b]$, suppose we have a uniform grid with nodes

$$
a=x_{\frac{1}{2}}<x_{\frac{3}{2}}<x_{\frac{5}{2}}<\cdots<x_{N-\frac{1}{2}}<x_{N+\frac{1}{2}}=b .
$$

We denote $i$ th cell $\left[x_{i-\frac{1}{2}}, x_{i+\frac{1}{2}}\right]$ as $\tau_{i}$ for $i=1,2, \ldots, N$. Its cell center is $x_{i}=\frac{1}{2}\left(x_{i-\frac{1}{2}}+x_{i+\frac{1}{2}}\right)$, and its cell size is $h_{i}=x_{i+\frac{1}{2}}-x_{i-\frac{1}{2}}$. The cell average of a function $u(x)$ over $\tau_{i}$ is then

$$
\bar{u}_{i}=\frac{1}{\Delta x_{i}} \int_{x_{i-\frac{1}{2}}}^{x_{i+\frac{1}{2}}} u(x) d x, \quad i=1,2, \ldots, N .
$$

For each cell $\tau_{i}$, our goal is to reconstruct a piecewise polynomial approximation $\widetilde{u}_{i}(x)$ of degree at most $p-1$, such that it approximates $u(x)$ to $p$ th order accuracy within $\tau_{i}$, i.e.,

$$
\widetilde{u}_{i}(x)=u(x)+\mathcal{O}\left(h^{p}\right), \quad x \in \tau_{i}, \quad i=1, \ldots, N,
$$

where $h=\min \left\{h_{i} \mid 1 \leq i \leq N\right\}$.

To find such a polynomial, a WENO scheme first selects $p$ sub-stencils about $\tau_{i}$, each containing $p$ cells. Consider a particular sub-stencil

$$
S_{j}(i)=\left\{\tau_{i-j}, \ldots, \tau_{i-j+p-1}\right\},
$$

and let $\phi_{i, j}(x)$ be a polynomial approximation of $u$ over $S_{j}(i)$, obtained by requiring the integral of $\phi_{i, j}(x)$ over each cell in the sub-stencil to be equal to that of $u_{i}(x)$. If the $p$ th derivative of $u$ is bounded over the sub-stencil $S_{j}(i)$, then $\phi_{i, j}(x)$ satisfies $(7)$. However, if $u(x)$ has discontinuities within the sub-stencil, then $\phi_{i, j}(x)$ may be oscillatory. The WENO scheme then constructs a non-oscillatory approximation by taking a convex combination of $\phi_{i, j}(x)$

$$
\widetilde{u}_{i}(x)=\sum_{j} \omega_{j} \phi_{i, j}(x)
$$

where $\omega_{j}=\alpha_{j} / \sum_{k=0}^{p-1} \alpha_{k}$ and is chosen such that $\omega_{j}$ approaches zero for sub-stencils with discontinuities. A typical choice of $\alpha_{j}$ is

$$
\alpha_{j}=d_{j} /\left(\epsilon+\beta_{j}\right)^{2}
$$


where $d_{j}$ is a nonnegative coefficient such that

$$
\widetilde{u}_{i}(x)=\sum_{j=0}^{p-1} d_{j} \phi_{i, j}(x)=u\left(x_{i+\frac{1}{2}}\right)+\mathcal{O}\left(h^{2 p-1}\right) .
$$

The parameter $\epsilon$ is a small parameter, such as $\epsilon=10^{-6}$, introduced to avoid instability due to division by zero or too small a number. The non-negativity of $d_{i}$ is important for stability purposes. The $\beta_{j}$ is the smoothness indicator. If $u(x)$ is smooth over the sub-stencil $S_{j}(i)$, then $\beta_{j}=\mathcal{O}\left(h^{2}\right)$; otherwise, $\beta_{j}=\mathcal{O}(1)$. A typical choice of $\beta_{j}$, as introduced in [2], is

$$
\beta_{j}=\sum_{k=1}^{p-1} \int_{x_{i-\frac{1}{2}}}^{x_{i+\frac{1}{2}}} h^{2 k-1}\left(\frac{\partial^{k} \phi_{j}(x)}{\partial x^{k}}\right)^{2} d x,
$$

where the $h^{2 k-1}$ term is introduced to make $\beta_{j}$ independent of the grid resolution. For example, in the simplest case where $p=2$,

$$
\begin{aligned}
& \beta_{0}=\left(\bar{u}_{i+1}-\bar{u}_{i}\right)^{2}, \\
& \beta_{1}=\left(\bar{u}_{i}-\bar{u}_{i-1}\right)^{2} .
\end{aligned}
$$

Alternative smoothness indicators have been proposed in [11, 12, 26, 27, 28].

\subsection{WENO Schemes on 2-D and 3-D Structured Meshes}

Originally developed in 1-D, the WENO schemes can be generalized to structured meshes in 2-D and 3 -D. Here, we give a brief overview of the reconstructions in $2-\mathrm{D}$, which generalize to $3-\mathrm{D}$ in a relatively straightforward manner.

Consider an $N_{x}$-by- $N_{y}$ structured grid, and let $\tau_{i j}$ denote the cell $(i, j)$ in the grid, where $i=1,2, \ldots, N_{x}$ and $j=1,2, . ., N_{y}$. Suppose the cell averages of a function $u(x, y)$,

$$
\bar{u}_{i j}=\frac{1}{\Delta x_{i} \Delta y_{j}} \int_{y_{j-\frac{1}{2}}}^{y_{j+\frac{1}{2}}} \int_{x_{i-\frac{1}{2}}}^{x_{i+\frac{1}{2}}} u(x, y) d x d y,
$$

are given. We would like to find

$$
\widetilde{u}_{i j}(x, y)=\sum_{r=0}^{p-1} \sum_{s=0}^{p-1} a_{r s} x^{r} y^{s},
$$

where $a_{r s}$ are the coefficients to be determined so that $\widetilde{u}_{i j}$ approximates $u$ to $p$ th order accuracy over $\tau_{i j}$.

Similar to the 1-D case, a WENO scheme first constructs polynomial approximations over a selection of sub-stencils and then computes a convex combination of these approximations. Consider a particular sub-stencil

$$
S_{l m}(i, j)=\left\{\tau_{I J}: i-l \leq I \leq i-l+p-1, j-m \leq J \leq j-m+p-1\right\} .
$$

Let $\phi_{i j l m}(x, y)$ denote a polynomial reconstruction of $u$ over $S_{l m}(i, j)$, whose integration over each cell in $S_{l m}(i, j)$ is equal to the given cell average. The function $\phi_{i j l m}(x, y)$ approximates $u(x, y)$ over $\tau_{i j}$ to $p$ th order accuracy for smooth functions. To obtain a non-oscillatory reconstruction for non-smooth functions, the WENO scheme computes $\widetilde{u}_{i j}$ as

$$
\widetilde{u}_{i j}(x, y)=\sum_{l} \sum_{m} \omega_{l m} \phi_{i j l m}(x, y) .
$$

The weights $\omega_{l m}$ are chosen so that the order of accuracy of $\widetilde{u}_{i j}$ is maximized for smooth functions, and then further augmented based on similar smoothness indicators as in 1-D, so that the weights would approach zero for the sub-stencils with discontinuities. For stability, it is important that the weights are nonnegative, which imposes some constraints to the selection of stencils. For more detail, see $[2,29,6]$. 


\subsection{ENO and WENO Schemes on Unstructured Meshes}

Besides structured meshes, WENO can also be generalized to unstructured meshes. Typically, these schemes are also constructed from some convex combination of lower-order schemes on sub-stencils. However, compared to structured meshes, it is much more challenging to construct stable WENO schemes on unstructured meshes, because it is difficult to satisfy the convexity requirement. Some WENO schemes have been proposed for 2-D $[5,7,29,6]$ and 3-D $[16,8,30,9,10]$. Below, we briefly review these generalizations, focusing on three different types.

The first type of WENO reconstruction, as proposed in [5], uses a combination of high-order polynomials computed from low-order polynomials over sub-stencils. For example, to obtain a third-order reconstruction, the scheme would first construct linear approximations over several sub-stencils by requiring the cell averages of the polynomials to be equal to the given cell averages, and then compute a quadratic polynomial from a weighted average of these linear polynomials. This technique works for unstructured meshes, even for meshes with mixed types of elements. However, depending on the mesh, the weighted average may not form a convex combination, and the linear system for calculating the weights may be ill-conditioned.

The second type of WENO reconstruction is similar to the first type, except that it compromises the order of accuracy of the convex combination, by allowing convex combination to be the same degree polynomials as those for the sub-stencils. Compared to the first type, this construction is less sensitive to mesh quality than the first type at the cost of lower accuracy. Therefore, it is often used as a fallback of the first type for robustness [19].

The third type of WENO reconstruction builds the reconstructions in a hierarchical fashion. An example is the approach in [29], which first finds the smoothest linear reconstructions over the first-layer three-cell stencils, and then use these linear reconstructions to build quadratic reconstructions over the second-layer stencils. This approach can be applied iteratively to construct higher-order reconstructions. However, as shown in [29], the accuracy of the reconstruction may not improve as the degree of the polynomial increases, especially near boundaries.

Besides the above WENO schemes, we also note some recent development of the Central ENO (CENO) schemes [31, 21], based on least squares approximations. CENO schemes require limiters for linear reconstructions to preserve monotonicity. Our proposed WLS-ENO schemes differ from the WENO and CENO reconstructions, in that the WLS-ENO schemes utilize a weighed least squares formulation, do not require limiters, and are insensitive to mesh quality due to adaptive stencils.

\section{Weighted-Least-Squares Based ENO Schemes}

In this section, we propose a new class of essentially non-oscillatory schemes, referred to as $W L S$-ENO. In the context of finite volume methods, these schemes reconstruct a function $u(\boldsymbol{x})$ over each cell, given the cell averages of $u$, denoted by $\bar{u}$, for all the cells. For each point along cell boundaries, the reconstructions then provide two values, $u^{-}$and $u^{+}$, which can then be used in (3) to calculate fluxes. Unlike WENO schemes, the WLS-ENO does not use weighted averaging of functional approximations over sub-stencils. Instead, it computes the reconstruction over each cell based on weighted least squares with an adaptive stencil. It can achieve optimal accuracy for smooth functions, stability around discontinuities, and insensitivity to mesh quality. We will present the derivations of WLS-ENO schemes based on Taylor series expansion, as well as their implementations in 1-D, 2-D and 3-D over structured and unstructured meshes.

\subsection{WLS-ENO Schemes in 1-D}

We first derive the WLS-ENO schemes in 1-D. Suppose we are given a grid

$$
a=x_{\frac{1}{2}}<x_{\frac{3}{2}}<x_{\frac{5}{2}}<\cdots<x_{N-\frac{1}{2}}<x_{N+\frac{1}{2}}=b,
$$

and the cell averages $\bar{u}_{i}$ of a function $u(x)$ over each cell $\tau_{i}, i=1,2, \ldots, N$. For generality, we assume the grid is non-uniform, with varying cell sizes. Below, we first describe how to reconstruct $u$ from $\bar{u}_{i}$ for smooth functions, and then augment the method to handle discontinuities. 


\subsubsection{WLS-Based Reconstruction for Smooth Functions}

Without a loss of generality, let us consider the reconstruction of $u$ over $\tau_{i}$ at its boundary point $x_{i+\frac{1}{2}}$. To achieve $p$ th order accuracy, we need to construct a polynomial approximation of degree at least $p-1$. We choose a stencil with $n$ cells to perform the reconstruction, where $n \geq p$.

Suppose there are $l$ cells to the left of $\tau_{i}$ in the stencil. The full stencil is given by the set

$$
S(i)=\left\{\tau_{i-l}, \ldots, \tau_{i-l+n-1}\right\} .
$$

From Taylor series expansion, we can approximate function $u(x)$ at point $x_{i+\frac{1}{2}}$ to $p$ th order accuracy by

$$
u(x)=\sum_{k=0}^{p-1} \frac{u^{(k)}\left(x_{i+\frac{1}{2}}\right)}{k !}\left(x-x_{i+\frac{1}{2}}\right)^{k}+\mathcal{O}\left(h^{p}\right),
$$

where $h$ denotes the average edge lengths. The cell average over $\tau_{j}$ in the stencil can be approximated by

$$
\begin{aligned}
\bar{u}_{j} & =\sum_{k=0}^{p-1} \frac{u^{(k)}\left(x_{i+\frac{1}{2}}\right)}{k !\left(x_{j+\frac{1}{2}}-x_{j-\frac{1}{2}}\right)} \int_{x_{j-\frac{1}{2}}}^{x_{j+\frac{1}{2}}}\left(x-x_{i+\frac{1}{2}}\right)^{k} d x+\mathcal{O}\left(h^{p}\right) \\
& =\sum_{k=0}^{p-1} \frac{u^{(k)}\left(x_{i+\frac{1}{2}}\right)}{(k+1) !}\left[\left(x_{j+\frac{1}{2}}-x_{i+\frac{1}{2}}\right)^{k+1}-\left(x_{j-\frac{1}{2}}-x_{i+\frac{1}{2}}\right)^{k+1}\right]+\mathcal{O}\left(h^{p}\right) .
\end{aligned}
$$

Given $\bar{u}_{j}$ at the $j$ th cells in $S(i)$, we then construct an $n \times p$ linear system

$$
A v \approx \bar{u},
$$

where

$$
a_{J K}=\frac{1}{K !}\left[\left(x_{i-l+J-\frac{1}{2}}-x_{i+\frac{1}{2}}\right)^{K}-\left(x_{i-l+J-\frac{3}{2}}-x_{i+\frac{1}{2}}\right)^{K}\right]
$$

for $J \equiv j+l+1-i \in[1, n]$ and $K \equiv k+1 \in[1, p], \overline{\boldsymbol{u}}$ is composed of the cell averages $\bar{u}_{j}$, and $\boldsymbol{v}$ is composed of the derivative of function $u$ at $x_{i+\frac{1}{2}}$, i.e., $v_{K}=u^{(k)}\left(x_{i+\frac{1}{2}}\right)$.

Eq. (21) in general is a rectangular linear system, and we can solve it using a weighted least squares formulation. In particular, we assign a different weight to each cell. Let $\boldsymbol{W}$ denote a diagonal matrix containing these weights. The problem can be written in matrix form as

$$
\min \|\boldsymbol{W} \boldsymbol{A} \boldsymbol{v}-\boldsymbol{W} \overline{\boldsymbol{u}}\|_{2} .
$$

The weights allow us to assign different priorities to different cells. For example, we may give higher weights to the cells closer to $I_{i}$. We solve this weighted least squares problem using QR factorization with column pivoting, as we will describe in more detail in Section 3.3. Since the method is derived based on Taylor series expansions directly, this WLS-based reconstruction can deliver the same order of accuracy as interpolationbased schemes for smooth functions, as we demonstrate in Section 4.1. For discontinuous functions, these weights can also allow us to suppress the influence of cells close to discontinuities, as we discuss next.

\subsubsection{WLS-ENO for Discontinuous Functions}

To apply WLS-ENO schemes to discontinuous functions, we modify the weighting matrix $\boldsymbol{W}$ in (23). The main idea is to assess the smoothness of the function within each cell of the stencil, and then define the weights correspondingly. By letting the weights be far smaller for the cells near discontinuities than those away from discontinuities, we can then effectively suppress oscillations.

We first construct a non-smoothness indicator of the function, analogous to those used in WENO schemes. Specifically, for the $j$ th cell in the stencil for $I_{i}$, with $j=i-l+J$, we can define the indicator for cell $I_{j}$ as

$$
\beta_{j}=\left\{\begin{array}{ll}
\left(\bar{u}_{j}-\bar{u}_{i}\right)^{2}+\epsilon h^{2} & j \neq i \\
\min \left\{\beta_{j-1}, \beta_{j+1}\right\} & j=i
\end{array},\right.
$$


where $\epsilon$ is a small constant, such as $\epsilon=10^{-2}$, introduced to avoid the indicator being too close to zero, and $h$ is some measure of average edge length. Note that $\beta_{j}=\mathcal{O}\left(h^{2}\right)$ if $u$ is smooth at $\tau_{j}$ and $\beta_{j}=\mathcal{O}(1)$ near discontinuities. Therefore, it captures the non-smoothness of the function. We therefore refer to $\beta$ as a non-smoothness indicator, although its counterpart in WENO is called the "smoothness indicator."

We then define the weights based on $\beta_{j}$. To suppress oscillations, it is desirable to use smaller weights for cells at discontinuities. Therefore, we make the weights in $\boldsymbol{W}$ to be inversely proportional to $\beta_{j}$ when $j \neq i$, and make the value larger for $\beta_{i}$. Specifically, we choose

$$
\omega_{j}=\left\{\begin{array}{ll}
1 / \beta_{j} & j \neq i \\
\alpha / \beta_{j} & j=i
\end{array},\right.
$$

where $\alpha>1$, such as $\alpha=1.5$. It is easy to see that $\omega_{j}$ is $\mathcal{O}\left(1 / h^{2}\right)$ if the function is smooth around cell $i$ and $\omega_{q}=\mathcal{O}(1)$ if the function is discontinuous in the cell. After computing the weights, we substitute them into (23) to compute the reconstruction. Note that unlike the weights in WENO, we do not need to normalize the weights by dividing them by the sum of the weights. As we will demonstrate in Section 4.1, this approach effectively suppresses the oscillations near discontinuities, similar to WENO schemes.

\subsection{Generalization of WLS-ENO Schemes to 2-D and 3-D}

The WLS-ENO reconstruction can be generalized to 2-D and 3-D, for evaluating the values at quadrature points along the cell boundaries. Similar to 1-D, we derive the higher-dimensional version of the linear system (21) over each cell based on Taylor series expansion, and then solve it based on weighted least squares.

Let us first consider the scheme in 2-D. First, we choose $n$ cells with index $i_{1}, i_{2}, \ldots, i_{n}$ as the stencil for cell $\tau_{i}$. Let $\left(x_{i}, y_{i}\right)$ denote its centroid. From the 2-D Taylor series expansion, we can approximate $u(x, y)$ about $\left(x_{i}, y_{i}\right)$ to $p$ th order accuracy by

$$
u(x, y)=\sum_{q=0}^{p-1} \sum_{k, l \geq 0}^{k+l=q} \frac{\partial^{q} u\left(x_{i}, y_{i}\right)}{\partial x^{k} \partial y^{l}} \frac{\left(x-x_{i}\right)^{k}\left(y-y_{i}\right)^{l}}{k ! l !}+\mathcal{O}\left(\|\delta\|^{p}\right),
$$

where $\delta=\max \left\{\left|x-x_{i}\right|,\left|y-y_{i}\right|\right\}$. Thus, the cell averages over the $j$ th cell $\tau_{j}$ can be approximated by

$$
\bar{u}_{j}=\frac{1}{k ! l !\left|\tau_{j}\right|} \sum_{q=0}^{p-1} \sum_{k, l \geq 0}^{k+l=q} \frac{\partial^{q} u\left(x_{i}, y_{i}\right)}{\partial x^{k} \partial y^{l}} \iint_{\tau_{j}}\left(x-x_{i}\right)^{k}\left(y-y_{i}\right)^{l} d x d y+\mathcal{O}\left(\|\delta\|^{p}\right)
$$

for $j=i_{1}, \ldots, i_{n}$, where $\left|\tau_{j}\right|$ denotes the area of $\tau_{j}$. Therefore, we obtain $n$ equations from the $n$ cells in the stencil about $\tau_{i}$, which can then be solved using the weighted least squares formulation (23).

The construction in 3-D is based on the 3-D Taylor series expansion about a centroid $\left(x_{i}, y_{i}, z_{i}\right)$ of the $i$ th cell $\tau_{i}$,

$$
u(x, y, z)=\sum_{q=0}^{p-1} \sum_{k, l, m \geq 0}^{k+l+m=q} \frac{\partial^{q} u\left(x_{i}, y_{i}, z_{i}\right)}{\partial x^{k} \partial y^{l} \partial z^{m}} \frac{\left(x-x_{i}\right)^{k}\left(y-y_{i}\right)^{l}\left(z-z_{i}\right)^{m}}{k ! l ! m !}+\mathcal{O}\left(\|\delta\|^{p}\right),
$$

where $\delta=\max \left\{\left|x-x_{i}\right|,\left|y-y_{i}\right|,\left|z-z_{i}\right|\right\}$. Then the cell averages over the $j$ th cell $\tau_{j}$ in the stencil for $\tau_{i}$ can be approximated by

$$
\bar{u}_{j}=\frac{1}{k ! l ! m !\left|\tau_{j}\right|} \sum_{q=0}^{p-1} \sum_{k, l, m \geq 0}^{k+l+m=q} \frac{\partial^{q} u\left(x_{i}, y_{i}, z_{i}\right)}{\partial x^{k} \partial y^{l} \partial z^{m}} \iiint_{\tau_{j}}\left(x-x_{i}\right)^{k}\left(y-y_{i}\right)^{l}\left(z-z_{i}\right)^{m} d x d y d z+\mathcal{O}\left(\|\delta\|^{p}\right)
$$

for $j=i_{1}, \ldots, i_{n}$, where $\left|\tau_{j}\right|$ denotes the volume of $\tau_{i}$. We can then solve the resulting least squares problem using weighted least squares.

To determine the weights in (23), we define the non-smoothness indicator $\beta_{j}$ similar to (24), and then define the weights $\omega_{j}$ as in (25). As in 1-D, it is easy to show that $\beta_{j}=\mathcal{O}\left(h^{2}\right)$ if $u$ is smooth at $\tau_{j}$ and $\beta_{j}=\mathcal{O}(1)$ near discontinuities, and therefore the weights can effectively suppress the effect of cells near discontinuities. After solving the linear system and obtaining the polynomial approximation, we can then evaluate $u(x, y)$ at the quadrature points along the boundary for computing the numerical fluxes.

One remaining question is the selection of the stencils. For the reconstruction over triangular meshes in 2-D, we adopt the strategy in [32] to define $k$-ring neighbor cells, with $1 / 2$-ring increments: 
- The $1 / 2$-ring neighbor cells are the cells that share an edge with a cell.

- The 1-ring neighbor cells of a cell are those that share at least one vertex with the center cell.

- For any positive integer $k$, the $(k+1)$-ring neighborhood of a cell is the union of $k$-ring neighborhood and 1-ring neighbors of its $k$-ring neighbor cells. The $(k+1 / 2)$-ring neighborhood is the union of $k$-ring neighborhood and $1 / 2$-ring neighbors of the $k$-ring neighbor cells.

Figure 1 illustrates the neighborhood definitions up to 2 rings. The $1 / 2$-ring increment allows finer granularity in the increment of the stencil sizes.

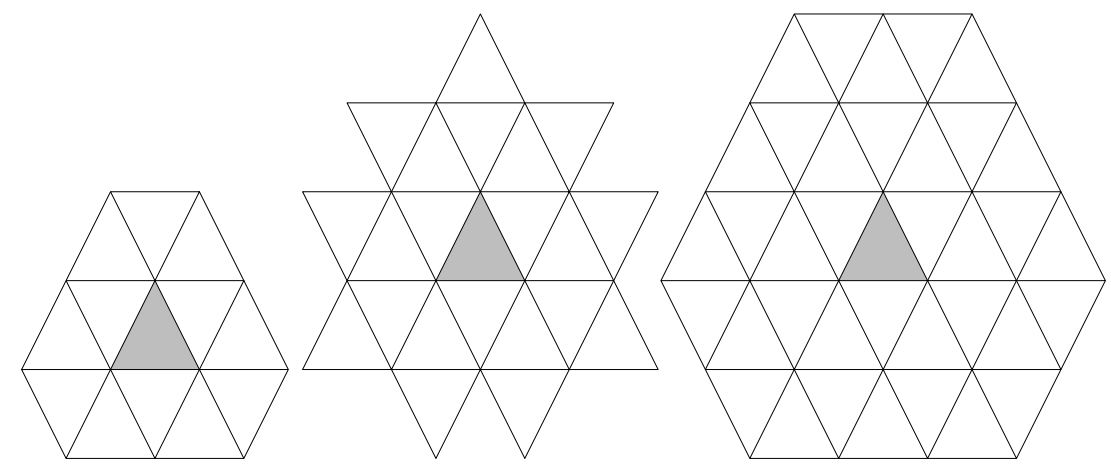

Figure 1: Examples of 1-ring, 1.5-ring and 2-ring neighborhood of a triangle.

For the reconstruction over tetrahedral meshes in $3-\mathrm{D}$, the standard $k$-ring neighbors grow very rapidly. To allow finer granularity, we define $k$-ring neighbor cells with $1 / 3$-ring increments, similar to those defined in [33]:

- The $1 / 3$-ring neighbor cells of a cell are the cells that share at least one face with the center cell.

- The $2 / 3$-ring neighbor cells of a cell are the cells that share at least one edge with the center cell.

- The 1-ring neighbor cells of a cell are the cells that share at least one vertex with the center cell.

- For any positive integer $k$, the $(k+1 / 3)$-ring neighborhood of a cell is the union of the $k$-ring neighborhood and the $1 / 3$-ring neighbors of its $k$-ring neighbor cells. The $(k+2 / 3)$-ring neighborhood is the union of the $k$-ring neighborhood and the $2 / 3$-ring neighbors of the $k$-ring neighbor cells.

With the above definitions, we adaptively choose the $k$-ring neighborhood, so that the number of cells in a stencil is approximately equal to 1.5 to 2 times the number of coefficients in the Taylor polynomial. This adaptive strategy allows the WLS-ENO scheme to be less dependent on mesh quality than WENO schemes.

The above strategy works well in practice for most cases. However, on poor-quality meshes, some $k$-ring neighborhoods may be nearly one-sided, which may cause the least-squares approximation to be closer to extrapolation along some directions. This may cause the reconstructed value to fall beyond the maximum and minimum values of the cell averages in the neighborhood, and in turn lead to oscillations. This rarely happens in 1-D or 2-D, but we do observe it in practice for some 3-D meshes. This issue could be mitigated by using limiters, analogous to the approach in [34] for multidimensional reconstructions on unstructured meshes. However, we resolve the issue by adapting the stencil as follows. First, after the reconstruction, we check whether the reconstructed values are between the maximum and minimum values in the neighborhood. If not, we compute the plane that passes through the centroid $\boldsymbol{x}_{i}$ of $\tau_{i}$ and is orthogonal to $\nabla u$ at $\boldsymbol{x}_{i}$. Next, we select a subset of the cells from an enlarged stencil to ensure the new stencil is well balanced on the two sides of the plane. Because of the smoothing nature of least squares, we find that the new polynomial approximation typically falls within the range on balanced stencils.

\subsection{Implementation Details}

The WLS-ENO is different from the WENO schemes in terms of the stencil selection and the local linear systems, and therefore it requires different data structures and linear algebra techniques. Below, we address some of these implementation details. 


\subsubsection{Data Structure for Neighborhood Search}

To support the construction of stencils in 2-D and 3-D, we use an Array-based Half-Facet (AHF) data structure [35] to store the mesh information. In a $d$-dimensional mesh, the term facet refers to the $(d-1)$ dimensional mesh entities; that is, for 2-D meshes the facets are the edges, and for 3-D meshes the facets are the faces. The basis for the half-facet data structure is the idea that every facet in a manifold mesh is made of two half-facets oriented in opposite directions. We refer to these two half-facets as sibling half-facets. Half-facets on the boundary of the domain have no siblings. The half-facets are half-edges and half-faces in 2-D and 3-D, respectively. We identify each half-facet by a two tuple: the element ID and a local facet ID within the element. In 2-D, we store the element connectivity, sibling half-edges, and a mapping from each node to an incident half-edge. In 3-D, we store the element connectivity, sibling half-faces, and a mapping from each node to an incident half-face. This data structure allows us to do neighborhood queries for a node in constant time (provided the valance is bounded). For additional information about the data structure, see [35].

\subsubsection{Solution of Weighted Least Squares Problems}

The technique for solving the least squares problem (21) is QR factorization with column pivoting. Suppose the size of $\boldsymbol{A}$ is $m \times n$. To further improve the condition number of $\boldsymbol{A}$, we scale the columns of the matrix $\boldsymbol{W} \boldsymbol{A}$ and then solve the following problem instead:

$$
\min _{\boldsymbol{d}}\|\boldsymbol{W} \boldsymbol{A S \boldsymbol { d }}-\boldsymbol{W} \overline{\boldsymbol{u}}\|_{2} .
$$

Here, $\boldsymbol{W} \in \mathbb{R}^{m \times m}$ is the weighting matrix in $(23), \boldsymbol{d} \equiv \boldsymbol{S}^{-1} \boldsymbol{V}$, and $\boldsymbol{S}=\operatorname{diag}\left(1 /\left\|\tilde{\boldsymbol{a}}_{1}\right\|_{2}, 1 /\left\|\tilde{\boldsymbol{a}}_{2}\right\|_{2}, \ldots, 1 /\left\|\tilde{\boldsymbol{a}}_{n}\right\|_{2}\right)$, where $\tilde{\boldsymbol{a}}_{i}$ is the $i$ th column vector of $\boldsymbol{W} \boldsymbol{A}$. We perform QR factorization with column pivoting to the matrix $\boldsymbol{W A S}$,

$$
W A S E=Q R,
$$

where $\boldsymbol{Q} \in \mathbb{R}^{m \times n}$ is composed of orthonormal columns, $\boldsymbol{R} \in \mathbb{R}^{n \times n}$ is upper triangular, and $\boldsymbol{E}$ is chosen so that the diagonal entries in $\boldsymbol{R}$ are in descending order. If $\boldsymbol{W} \boldsymbol{A} \boldsymbol{S}$ has full rank, then its pseudoinverse is

$$
(\boldsymbol{W} \boldsymbol{A S})^{+}=\boldsymbol{E} \boldsymbol{R}^{-1} \boldsymbol{Q}^{T} .
$$

Otherwise, we compute the pseudoinverse as

$$
(\boldsymbol{W} \boldsymbol{A} \boldsymbol{S})^{+}=\boldsymbol{E}_{1: k, 1: r} \boldsymbol{R}_{1: r, 1: r}^{-1}\left(\boldsymbol{Q}_{1: m, 1: r}\right)^{T},
$$

where $r$ is the numerical rank of $\boldsymbol{R}$. In this way, we can truncate the higher order terms in $\boldsymbol{W} \boldsymbol{A} \boldsymbol{S}$ for best-possible accuracy whenever possible.

\section{Accuracy and Stability of WLS-ENO}

In this section, we analyze the accuracy of WLS-ENO, as well as its stability in the context of solving hyperbolic conservation laws.

\subsection{Accuracy}

First, we analyze the WLS-ENO schemes and show that they can achieve the expected order of accuracy for smooth functions.

Theorem 1. Given a mesh with a smooth function $f$. Let $\boldsymbol{W}$ be a diagonal matrix containing all the weights for the cells. $\boldsymbol{A}$ and $\boldsymbol{S}$ are the matrices in (30). Suppose the cell average of $f$ is approximated with an error $\mathcal{O}\left(h^{p}\right)$ and the matrix $\boldsymbol{W} \boldsymbol{A S}$ has a bounded condition number. The degree- $(p-1)$ cell average weighed least squares fitting approximates qth order derivatives of function $f$ to $\mathcal{O}\left(h^{p-q}\right)$.

For simplicity, we only prove the theorem in 2-D. The analysis also applies to 1-D and 3-D. 
Proof. The 2-D Taylor series expansion about the point $\left(x_{i}, y_{i}\right)$ reads

$$
f(x, y)=\sum_{q=0}^{p-1} \sum_{j, k \geq 0}^{j+k=q} \frac{f_{j k}}{j ! k !}\left(x-x_{i}\right)^{j}\left(y-y_{i}\right)^{k}+\mathcal{O}\left(\|\delta \boldsymbol{x}\|^{p}\right),
$$

where $\delta \boldsymbol{x}=\left[x-x_{i}, y-y_{i}\right]^{T}$. The cell average of $f(x, y)$ over some cell $\tau_{i}$ can be written as

$$
\frac{1}{\left|\tau_{i}\right|} \iint_{\tau_{i}} f(x, y) d x d y=\frac{1}{\left|\tau_{i}\right|} \sum_{q=0}^{p-1} \sum_{j, k \geq 0}^{j+k=q} \frac{f_{j k}}{j ! k !} \iint_{\tau_{i}}\left(x-x_{i}\right)^{j}\left(y-y_{i}\right)^{k} d x d y+\mathcal{O}\left(\|\delta \boldsymbol{x}\|^{p}\right) .
$$

Let $\boldsymbol{v}$ denote the exact derivatives of function $f, \tilde{\boldsymbol{v}}$ the numerical solution from the WLS fitting. Let $\boldsymbol{r}=\overline{\boldsymbol{u}}-\boldsymbol{A} \boldsymbol{v}$. By assumption, each component of $\boldsymbol{r}$ is $\mathcal{O}\left(\|\delta \boldsymbol{x}\|^{p}\right)$. The error of coefficients has the relationship $\boldsymbol{W} \boldsymbol{A}(\tilde{\boldsymbol{v}}-\boldsymbol{v}) \approx \boldsymbol{W} \boldsymbol{r}$. The error of $\boldsymbol{d}$ can then be written as

$$
\boldsymbol{W} \boldsymbol{A} \boldsymbol{S} \delta \boldsymbol{d} \approx \boldsymbol{W r} .
$$

By solving this least squares problem, we have $\delta \boldsymbol{d}=(\boldsymbol{W} \boldsymbol{A S})^{+} \boldsymbol{W} \boldsymbol{r}$. Since the function $f$ is smooth, all the diagonal entries in $\boldsymbol{W}$ are $\mathcal{O}\left(1 /\|\delta \boldsymbol{x}\|^{2}\right)$. Under the assumption that $\boldsymbol{W} \boldsymbol{A} \boldsymbol{S}$ has a bounded condition number $\kappa$, all the component of $\delta \boldsymbol{d}$ are $\mathcal{O}\left(\kappa\|\delta \boldsymbol{x}\|^{p-2}\right)$. For a $q$ th order partial derivative of function $f$, the corresponding column in $\boldsymbol{W} \boldsymbol{A}$ is $\mathcal{O}\left(\|\delta \boldsymbol{x}\|^{q-2}\right)$, so is the 2-norm of the column. Therefore, the $q$ th order derivatives of function $f$ are approximated to $\mathcal{O}\left(h^{p-q}\right)$.

From this theorem, we can conclude that the degree- $(p-1)$ WLS-ENO reconstruction delivers a $p$ th order accurate reconstruction for smooth functions.

\subsection{Stability of WLS-ENO for Hyperbolic Conservation Laws}

For the WLS-ENO to be practically useful, it is important that it enables a stable discretization for hyperbolic conservation laws, when coupled with a proper time-integration scheme. In the following, we analyze two fifth-order WLS-ENO schemes for a model problem in 1-D, based on a modified von Neumann stability analysis. We show that the WLS-ENO scheme has a larger stability region than the WENO scheme on structured meshes. We will further demonstrate its stability for 2-D and 3-D problems in Section 5.

\subsubsection{Model Problem in 1-D}

We consider one dimensional wave equation

$$
u_{t}+u_{x}=0, \quad x \in[0,1], \quad t>0
$$

with the periodic boundary condition

$$
u(x, 0)=u^{0}(x), \quad x \in[0,1] .
$$

Suppose we have a structured grid $0=x_{\frac{1}{2}}<x_{\frac{3}{2}}<\cdots<x_{N+\frac{1}{2}}=1$ with $x_{i}=i \Delta x$ and $\Delta x=1 / N$. For the finite volume method, we integrate the above wave equation and divide it by the length of the cell, and obtain

$$
\frac{d \bar{u}\left(x_{i}, t\right)}{d t}=-\frac{1}{\Delta x_{i}}\left(f\left(u\left(x_{i+\frac{1}{2}}, t\right)\right)-f\left(u\left(x_{i-\frac{1}{2}}, t\right)\right)\right)
$$

where

$$
\bar{u}\left(x_{i}, t\right)=\frac{1}{\Delta x_{i}} \int_{x_{i-\frac{1}{2}}}^{x_{i+\frac{1}{2}}} u(x, t) d x .
$$

We approximate (39) by the following conservative scheme

$$
\frac{d \bar{u}\left(x_{i}, t\right)}{d t}=-\frac{1}{\Delta x_{i}}\left(\hat{f}_{i+\frac{1}{2}}-\hat{f}_{i-\frac{1}{2}}\right),
$$


where the numerical flux $\hat{f}_{i+\frac{1}{2}}$ is replaced by the Lax-Friedrichs flux (3), with $\alpha=\max _{u}\left|f^{\prime}(u)\right|$. For this particular problem, $\hat{f}_{i+\frac{1}{2}}-\hat{f}_{i-\frac{1}{2}}=u_{i+\frac{1}{2}}^{-}-u_{i-\frac{1}{2}}^{-}$.

For time integration, we use a TVD Runge-Kutta method [1]. An $n$-stage Runge-Kutta method for the ODE $u_{t}=L(u)$ has the general form of

$$
\begin{aligned}
k_{0} & =u(t), \\
k_{i} & =\sum_{j=0}^{i-1}\left(\alpha_{i j} k_{j}+\beta_{i j} \Delta t L\left(k_{j}\right)\right), \quad i=1, \ldots, n,
\end{aligned}
$$

where $k_{i}$ denotes the intermediate solution after the $i$ th stage, and $u(t+\Delta t)=k_{n}$. A Runge-Kutta method is total variation diminishing (TVD) if all the coefficients $\alpha_{i j}$ and $\beta_{i j}$ are nonnegative. The CFL coefficient of such a scheme is given by

$$
c=\min _{i, k}\left\{\alpha_{i k} / \beta_{i k}\right\} .
$$

Specifically, we use the third-order TVD Runge-Kutta scheme, given by

$$
\begin{aligned}
k_{1} & =u+\Delta t L(u), \\
k_{2} & =\frac{3}{4} u+\frac{1}{4} k_{1}+\frac{1}{4} \Delta t L\left(k_{1}\right), \\
k_{3} & =\frac{1}{3} u+\frac{2}{3} k_{2}+\frac{2}{3} \Delta t L\left(k_{2}\right),
\end{aligned}
$$

for which the CFL coefficient is $c=1$.

\subsection{2. von Neumann Stability Analysis}

Based on the von Neumann stability analysis, the semi-discrete solution can be expressed by a discrete Fourier series

$$
\bar{u}_{j}(t)=\sum_{k=-N / 2}^{N / 2} \hat{u}_{k}(t) e^{i \omega_{k} j \Delta x}, \quad \omega_{k} \in R .
$$

By the superposition principle, we can only use one term in the series for analysis

$$
\bar{u}_{j}(t)=\hat{u}_{k}(t) e^{i j \theta_{k}}, \quad \theta_{k}=\omega_{k} \Delta x
$$

where $k=-N / 2, \ldots, N / 2$. We assume that the numerical flux can be written in the following form

$$
\hat{f}_{i+\frac{1}{2}}-\hat{f}_{i-\frac{1}{2}}=u_{i+\frac{1}{2}}^{-}-u_{i-\frac{1}{2}}^{-}=z\left(\theta_{k}\right) \bar{u}_{i}
$$

where the complex function $z\left(\theta_{k}\right)$ is the Fourier symbol.

Let $\bar{u}_{i}^{n}=\bar{u}_{i}\left(t^{n}\right)$ be the numerical solution at time level $t^{n}=n \Delta t$. We define the amplification factor $g$ by inserting (49) into the fully-discrete system and obtain

$$
\bar{u}_{i}^{n+1}=g\left(\hat{z}_{k}\right) \bar{u}_{i}^{n}, \quad \hat{z}_{k}=-\sigma z\left(\theta_{k}\right), \quad k=-N / 2, \ldots, N / 2,
$$

where $\sigma=\Delta t / \Delta x$. Therefore, the linear stability domain of an explicit time-stepping scheme is $S_{t}=$ $\{\hat{z}:|g(\hat{z})| \leq 1\}$. Also, we define the discrete spectrum $S$ of a spatial discretization scheme

$$
S=\left\{-z\left(\theta_{k}\right): \theta_{k} \in 0, \Delta \theta, 2 \Delta \theta, \ldots, 2 \pi\right\}, \quad \Delta \theta=2 \pi \Delta x .
$$

The stability limit is thus the largest CFL number $\widetilde{\sigma}$ such that the rescaled spectrum $\widetilde{\sigma} S$ lies inside the stability domain

$$
\tilde{\sigma} S \in S_{t} .
$$

For the third-order Runge-Kutta scheme, the amplification factor is given by

$$
g(\widetilde{z})=1+\widetilde{z}+\frac{1}{2} \widetilde{z}^{2}+\frac{1}{6} \widetilde{z}^{3} .
$$

To determine the boundary of the stability domain $\partial S_{t}=\{\widetilde{z}:|g(\widetilde{z})|=1\}$, we set $g(\widetilde{z})=e^{i \phi}$ and solve the following equation

$$
\widetilde{z}^{3}+3 \widetilde{z}^{2}+6 \widetilde{z}+6-6 e^{i \phi}=0 .
$$

The stability region is bounded by the solid blue curves in Figure 2 . 


\subsubsection{Fifth-Order WLS-ENO Scheme with Five Cells}

Let us first consider a fifth-order scheme for a cell $\tau_{i}$, with five cells

$$
S_{i}=\left\{\tau_{i-2}, \tau_{i-1}, \tau_{i}, \tau_{i+1}, \tau_{i+2}\right\},
$$

where $\tau_{i}$ is the interval $\left[x_{i-\frac{1}{2}}, x_{i+\frac{1}{2}}\right]$. For this stencil, the coefficient matrix in (21) is given by

$$
\boldsymbol{A}=\left(\begin{array}{ccccc}
1 & -\frac{5 \Delta x}{2} & \frac{19 \Delta x^{2}}{6} & -\frac{65 \Delta x^{3}}{24} & \frac{211 \Delta x^{4}}{120} \\
1 & -\frac{3 \Delta x}{2} & \frac{7 \Delta x^{2}}{6} & -\frac{15 \Delta x^{3}}{24} & \frac{31 \Delta x^{4}}{120} \\
1 & -\frac{\Delta x}{2} & \frac{\Delta x^{2}}{6} & -\frac{\Delta x^{3}}{24} & \frac{\Delta x^{4}}{120} \\
1 & \frac{\Delta x}{2} & \frac{\Delta x^{2}}{6} & \frac{\Delta x^{3}}{24} & \frac{\Delta x^{4}}{120} \\
1 & \frac{3 \Delta x}{2} & \frac{7 \Delta x^{2}}{6} & \frac{15 \Delta x^{3}}{24} & \frac{31 \Delta x^{4}}{120}
\end{array}\right) .
$$

Since $\boldsymbol{A}$ is nonsingular, the weights do not affect the solution. The solution is given by

$$
u_{i+\frac{1}{2}}^{-}=\frac{2}{60} \bar{u}_{i-2}-\frac{13}{60} \bar{u}_{i-1}+\frac{47}{60} \bar{u}_{i}+\frac{27}{60} \bar{u}_{i+1}-\frac{3}{60} \bar{u}_{i+2} .
$$

This is mathematically equivalent to the fifth-order WENO scheme without the nonlinear weights. Also, the flux reads

$$
u_{i+\frac{1}{2}}^{-}-u_{i-\frac{1}{2}}^{-}=-\frac{2}{60} \bar{u}_{i-3}+\frac{15}{60} \bar{u}_{i-2}-\frac{60}{60} \bar{u}_{i-1}+\frac{20}{60} \bar{u}_{i}+\frac{30}{60} \bar{u}_{i+1}-\frac{3}{60} \bar{u}_{i+2} .
$$

Substituting it into (49), we get

$$
z\left(\theta_{k}\right)=\frac{16}{15} \sin ^{6}\left(\frac{\theta_{k}}{2}\right)+i\left(-\frac{1}{6} \sin \left(2 \theta_{k}\right)+\frac{4}{3} \sin \left(\theta_{k}\right)+\frac{16}{15} \sin ^{5}\left(\frac{\theta_{k}}{2}\right) \cos \left(\frac{\theta_{k}}{2}\right)\right) .
$$

The discrete spectrum is shown in Figure 2(left).

Given the spectrum and the stability domain, the CFL number of this scheme can be computed by finding the largest rescaling parameter $\sigma$, so that the rescaled spectrum still lies in the stability domain. Using interval bisection, we find that the CFL number is $\sigma=1.44$ for the fifth-order five-cell scheme.

\subsubsection{Fifth-Order WLS-ENO Scheme with Seven Cells}

Next, let us consider a least-squares fitting for $\tau_{i}$ using seven cells

$$
S_{i}=\left\{\tau_{i-3}, \tau_{i-2}, \tau_{i-1}, \tau_{i}, \tau_{i+1}, \tau_{i+2}, \tau_{i+3}\right\} .
$$

The coefficient matrix in (21) is given by

$$
\boldsymbol{A}=\left(\begin{array}{ccccc}
1 & -\frac{7 \Delta x}{2} & \frac{37 \Delta x^{2}}{6} & -\frac{175 \Delta x^{3}}{24} & \frac{781 \Delta x^{4}}{120} \\
1 & -\frac{5 \Delta x}{2} & \frac{19 \Delta x^{2}}{6} & -\frac{65 \Delta x^{3}}{24} & \frac{211 \Delta x^{4}}{120} \\
1 & -\frac{3 \Delta x}{2} & \frac{7 \Delta x^{2}}{6} & -\frac{15 \Delta x^{3}}{24} & \frac{31 \Delta x^{4}}{120} \\
1 & -\frac{\Delta x}{2} & \frac{\Delta x^{2}}{6} & -\frac{\Delta x^{3}}{24} & \frac{\Delta x^{4}}{120} \\
1 & \frac{\Delta x}{2} & \frac{\Delta x^{2}}{6} & \frac{\Delta x^{3}}{24} & \frac{\Delta x^{4}}{120} \\
1 & \frac{3 \Delta x}{2} & \frac{7 \Delta x^{2}}{6} & \frac{15 \Delta x^{3}}{24} & \frac{31 \Delta x^{4}}{120} \\
1 & \frac{5 \Delta x}{2} & \frac{19 \Delta x^{2}}{6} & \frac{65 \Delta x^{3}}{24} & \frac{211 \Delta x^{4}}{120}
\end{array}\right) .
$$

If $\epsilon=0$, from (24) and (25), we obtain the weights

$$
w_{1} \approx \frac{1}{9 \Delta x^{2}}, \quad w_{2} \approx \frac{1}{4 \Delta x^{2}}, \quad w_{3} \approx \frac{1}{\Delta x^{2}}, \quad w_{4} \approx \frac{1.5}{\Delta x^{2}}, \quad w_{5} \approx \frac{1}{\Delta x^{2}}, \quad w_{6} \approx \frac{1}{4 \Delta x^{2}}, \quad w_{7} \approx \frac{1}{9 \Delta x^{2}} .
$$

Solving the weighted least squares system, we obtain the following scheme

$$
\begin{aligned}
u_{i+\frac{1}{2}}^{-}= & \frac{1226983}{9489680} \bar{u}_{i-3}-\frac{963431}{47447340} \bar{u}_{i-4}-\frac{13515169}{94894680} \bar{u}_{i-1}+\frac{66771}{87380} \bar{u}_{i}+ \\
& \frac{38388551}{94894680} \bar{u}_{i+1}-\frac{93404}{11861835} \bar{u}_{i+2}-\frac{348299}{31631560} \bar{u}_{i+3} .
\end{aligned}
$$


Therefore, the flux reads

$$
\begin{aligned}
u_{i+\frac{1}{2}}^{-}-u_{i-\frac{1}{2}}^{-}= & -\frac{1226983}{94894680} \bar{u}_{i-4}+\frac{630769}{18978936} \bar{u}_{i-3}+\frac{3862769}{31631560} \bar{u}_{i-2}-\frac{17205695}{18978936} \bar{u}_{i-1}+ \\
& \frac{6824951}{18978936} \bar{u}_{i}+\frac{13045261}{31631560} \bar{u}_{i+1}+\frac{59533}{18978936} \bar{u}_{i+2}-\frac{348299}{31631560} \bar{u}_{i+3 .} .
\end{aligned}
$$

Substituting it into (49), we get

$$
z\left(\theta_{k}\right)=p\left(\theta_{k}\right)+i q\left(\theta_{k}\right)
$$

where

$$
p\left(\theta_{k}\right)=-0.0129 \cos \left(4 \theta_{k}\right)+0.0222 \cos \left(3 \theta_{k}\right)+0.1253 \cos \left(2 \theta_{k}\right)-0.4942 \cos \left(\theta_{k}\right)+0.3596,
$$

and

$$
q\left(\theta_{k}\right)=0.0129 \sin \left(4 \theta_{k}\right)-0.0442 \sin \left(3 \theta_{k}\right)-0.1190 \sin \left(2 \theta_{k}\right)+1.3190 \sin \left(\theta_{k}\right) .
$$

The discrete spectrum is shown in Figure 2(right). The CFL number is computed in the same way as the above scheme. For this scheme with seven cells, we obtain $\sigma=1.67$. Therefore, the seven-cell fifthorder WLS-ENO scheme has a larger stability region than the five-cell counterpart, which is mathematically equivalent to the fifth-order WENO scheme without nonlinear weights.
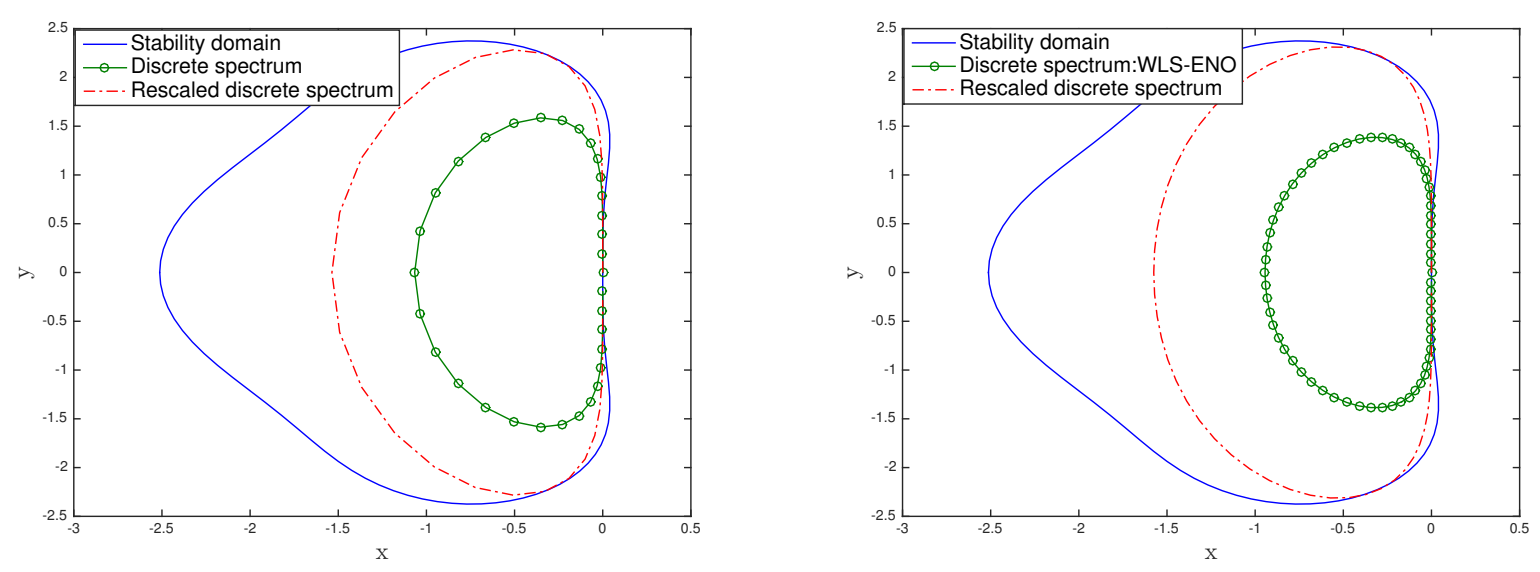

Figure 2: Rescaled spectra and stability domains of fifth-order WLS-ENO with five (left) and seven (right) cells.

\section{Numerical Results}

In this section, we present some numerical experiments of WLS-ENO over both structured and unstructured meshes in 1-D, 2-D, and 3-D, and compare it against WENO schemes when applicable. For all the PDEs, we will use third-order TVD Runge-Kutta for time integration.

\subsection{1-D Results}

We first show some results in 1-D, for the reconstruction of a piecewise smooth function as well as the solutions of PDEs, including a linear wave equation, Burgers' equation, and the Euler equations.

\subsubsection{Reconstruction of Discontinuous Functions}

We first test WLS-ENO for the reconstruction of a 1-D discontinuous function, given by

$$
v(x)=\left\{\begin{array}{cc}
\sin (\pi x) & 0 \leq x \leq 0.6 \\
\cos (\pi x) & 0.6<x \leq 1
\end{array} .\right.
$$


This function is discontinuous at $x=0.6$ but smooth everywhere else within the interval $[0,1]$. We performed grid convergence study under grid refinement, starting from an equidistant grid with 32 grid cells. For the WLS-ENO, we used degree-four polynomials over seven-cell stencil, which according to our theory should deliver fifth-order accuracy in smooth regions and fourth-order accuracy near discontinuities. As a point of reference, we also perform the reconstruction using the fifth-order WENO scheme, which is fifth-order accurate in smooth regions and third-order accurate near discontinuities. Figure 3 shows the $L_{\infty}$-norm error for the reconstructed values at the grid points that are one cell away from the discontinuity. It can be seen that both WLS-ENO and WENO delivered fifth-order accuracy, but WLS-ENO is more accurate. When including the grid points near discontinuities, as can be seen in Figure 4, WLS-ENO achieved fourthorder convergence, whereas the fifth-order WENO reduced to third order, as predicted by their respective theoretical analyses.

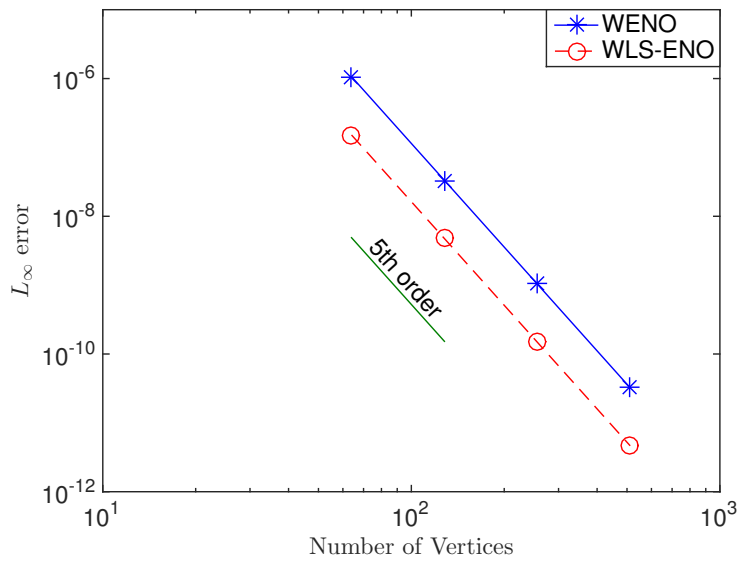

Figure 3: Convergence of fifth-order WENO and WLS-ENO away from discontinuity.

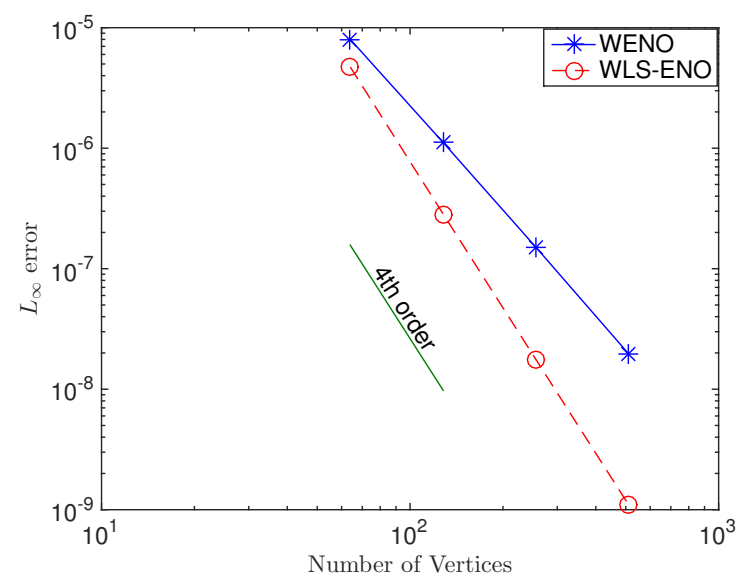

Figure 4: Convergence of fifth-order WENO and WLS-ENO near discontinuity.

\subsubsection{1-D Wave Equation}

To test the effectiveness of WLS-ENO for solving hyperbolic PDEs, we first solve a simple linear wave equation

$$
u_{t}+u_{x}=0, \quad-1 \leq x \leq 1,
$$

with periodic boundary conditions. Similar to the reconstruction problem, we use WLS-ENO with degreefour polynomials over seven cells. To assess the accuracy for smooth solutions, consider the smooth initial condition

$$
u(x, 0)=\sin (\pi x),
$$

for which the solution remains smooth over time. We assess the order of accuracy of the solutions at $t=1$ under grid refinement, and compare the errors against the fifth-order WENO. Figure 5 shows the results for uniform and non-uniform grids. For uniform grids, similar to the results of reconstruction, both WLS-ENO and WENO delivered fifth-order convergence under grid refinement, and the solution of WLS-ENO is more accurate. For non-uniform grids, we used the WENO reconstruction in [2], which converged at a slower rates, and was about an order of magnitude less accurate than WLS-ENO on the finest grid.

To demonstrate the accuracy and stability of WLS-ENO for discontinuous solutions, we change the initial condition to be a piecewise smooth function

$$
u(x, 0)=\left\{\begin{array}{ll}
\sin (\pi x) & -1 \leq x<-0.2 \cup 0.3<x \leq 1 \\
0.5 & -0.2 \leq x \leq 0.3
\end{array},\right.
$$

as shown in Figure 6(a). Figure 6(b) shows the solution at $t=0.5$ using WLS-ENO. The results show that the WLS-ENO scheme well preserved the sharp feature and maintained the non-oscillatory property. 


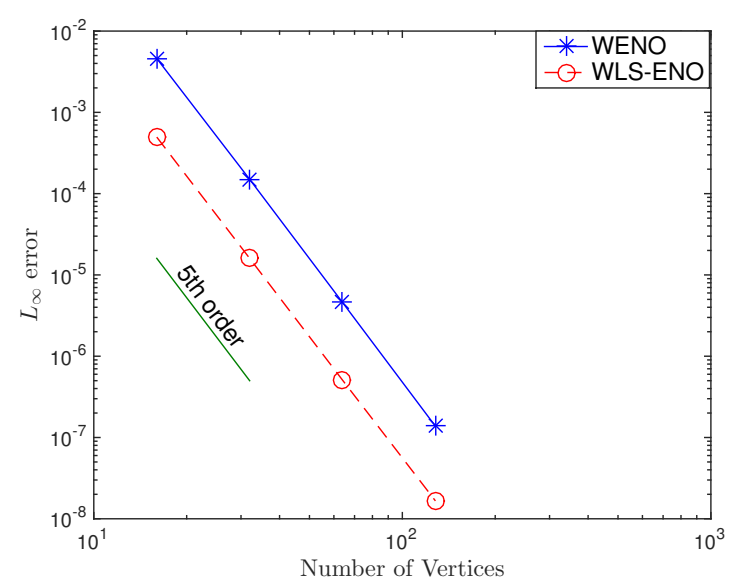

(a) Errors on uniform grids.

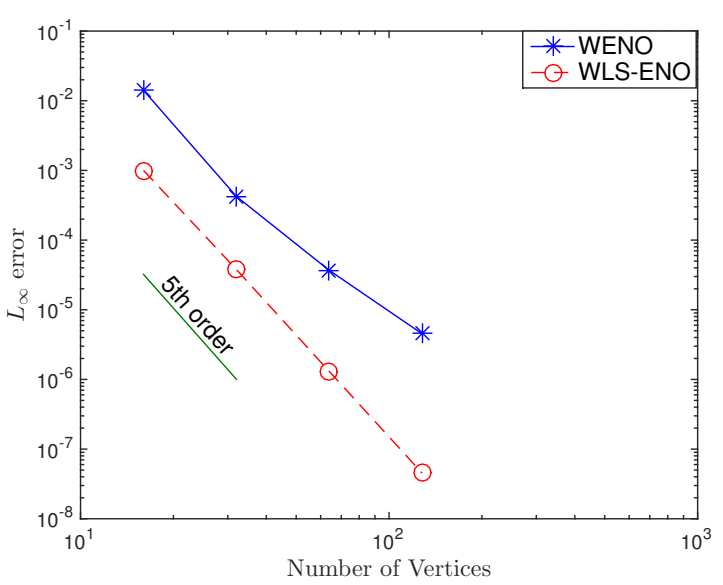

(b) Errors on non-uniform grids.

Figure 5: Convergence of fifth-order WENO and WLS-ENO for wave equation at $t=1$ on 1-D grids.

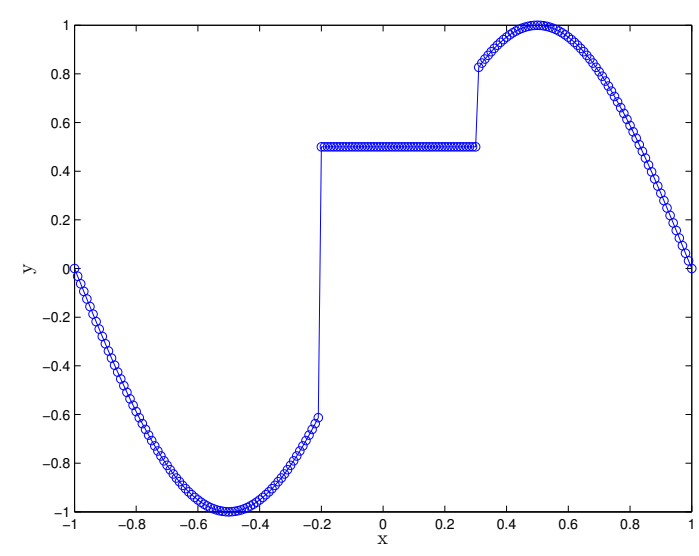

(a) Initial condition.

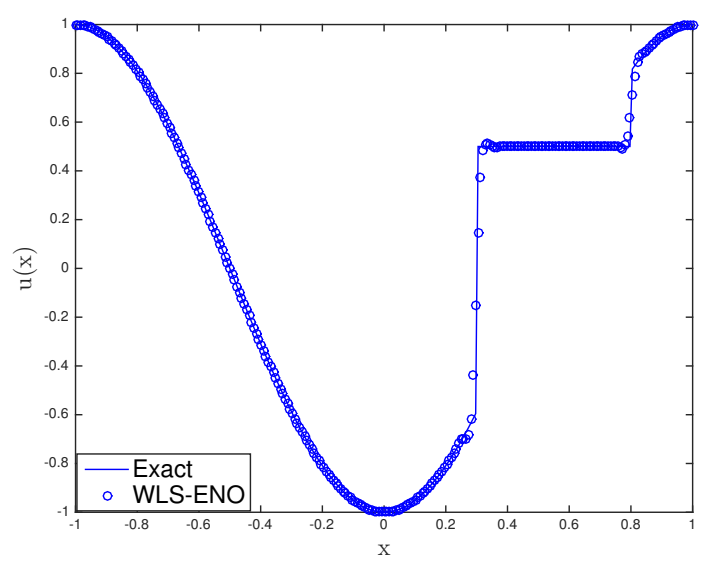

(b) Numerical solution at $t=0.5$.

Figure 6: Discontinuous initial condition (left) and numerical solution with fifth-order WLS-ENO (right) at $t=0.5$ for the linear wave equation.

\subsubsection{1-D Burgers' Equation}

Next, we test WLS-ENO with the 1-D Burgers' equation,

$$
\frac{\partial u}{\partial t}+u \frac{\partial u}{\partial x}=0,0 \leq x \leq 2 \pi
$$

with periodic boundary conditions and the initial condition

$$
u(x, 0)=0.3+0.7 \sin (x), 0 \leq x \leq 2 \pi .
$$

Although the initial condition is smooth, a discontinuity develops at time $t=1.4$. To assess the order of accuracy, Figure 7(a) shows the solutions from the fifth-order WLS-ENO, compared with the fifth-order WENO under grid refinement at time $t=1$, starting from a grid with 64 grid points. The results show that both WLS-ENO and WENO delivered fifth-order accuracy, while WLS-ENO is more accurate. Figure 7(b) shows the numerical solution from WLS-ENO overlaid on top of the exact solution at $t=1.4$. We can see that the WLS-ENO scheme approximated the solution very well. 


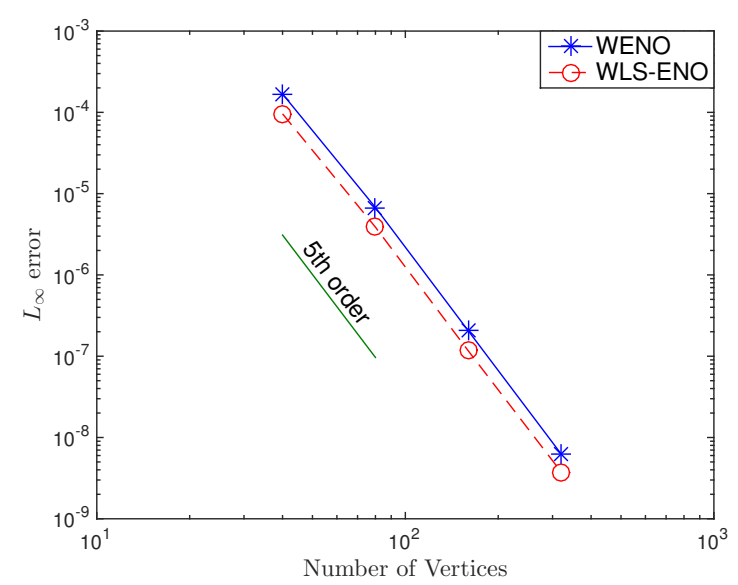

(a) Errors on uniform grids at $t=1$.

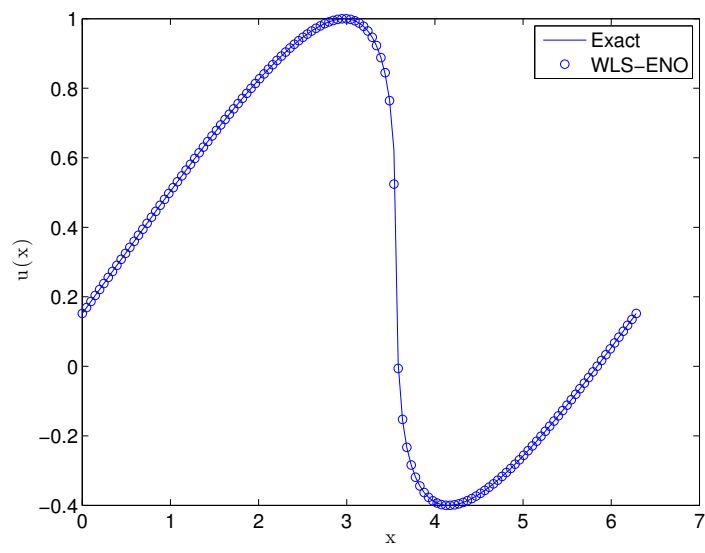

(b) Numerical and exact solution at $t=1.4$.

Figure 7: Comparison of WENO and WLS-ENO schemes for 1-D Burgers' equation at $t=1$ (left) and numerical solution with WLS-ENO (right) at $t=1.4$

\subsubsection{1-D Euler Equations}

The above tests demonstrate the accuracy and stability of WLS-ENO for 1-D benchmark problems. For a more realistic problem, we consider the 1-D Euler equations

$$
\left(\begin{array}{c}
\rho \\
\rho v \\
E
\end{array}\right)_{t}+\left(\begin{array}{c}
\rho v \\
\rho v^{2}+p \\
v(E+p)
\end{array}\right)_{x}=\mathbf{0},
$$

with the equation of state for ideal polytropic gas

$$
E=\frac{p}{\gamma-1}+\frac{1}{2} \rho v^{2}
$$

where $\rho$ denotes the gas density, $v$ the velocity, $p$ the pressure, $E$ the energy, and $\gamma=1.4$ a constant specific to air. We perform characteristic decomposition [36] and solve the conservation law characteristic-wise using the fifth-order WLS-ENO scheme on an unstructured (i.e., non-uniform) grid. In more detail, if we introduce the speed of sound $c$ by

$$
c=\sqrt{\frac{\gamma p}{\rho}}
$$

and enthalpy $H$ by

$$
H=\frac{E+p}{\rho},
$$

we have the eigenvalue decomposition for the Jacobian as

$$
R^{-1}(u) f^{\prime}(u) R(u)=\Lambda(u),
$$

where

$$
\begin{gathered}
f^{\prime}(u)=\left(\begin{array}{ccc}
0 & 1 & 0 \\
\left(\frac{\gamma-3}{2}\right) v^{2} & (3-\gamma) v & \gamma-1 \\
\left(\frac{\gamma-1}{2}\right)^{3}-v H & H-(\gamma-1) v^{2} & \gamma v
\end{array}\right), \\
\Lambda(u)=\left(\begin{array}{ccc}
v-c & \\
& v & \\
& & v+c
\end{array}\right),
\end{gathered}
$$

and

$$
R(u)=\left(\begin{array}{ccc}
1 & 1 & 1 \\
v-c & v & v+c \\
H-c v & \frac{1}{2} v^{2} & H+c v
\end{array}\right)
$$


Sod's Problem. We first compute the density, velocity and pressure with the initial condition given by Sod's problem [2]

$$
\left(\rho_{L}, v_{L}, p_{L}\right)=(1,0,1),\left(\rho_{R}, v_{R}, p_{R}\right)=(0.125,0,0.1) .
$$

Figure 8 shows the numerical solutions at $t=1$ compared against the exact solution. The results matched the exact solution very well and were non-oscillatory near discontinuities.

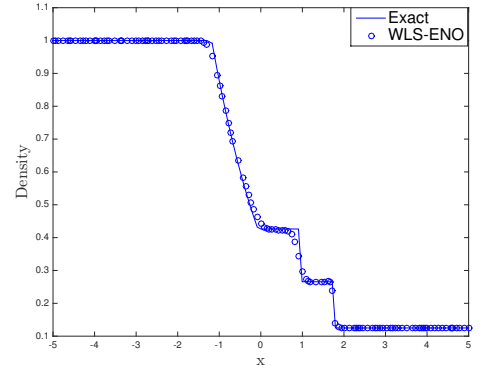

(a) Density.

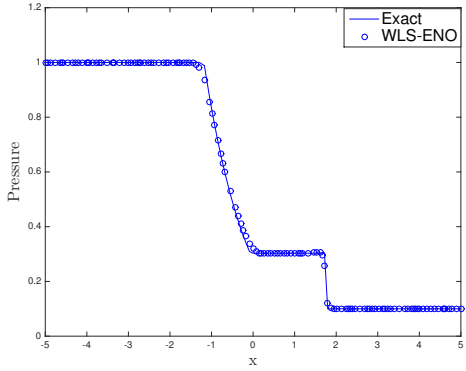

(b) Pressure.

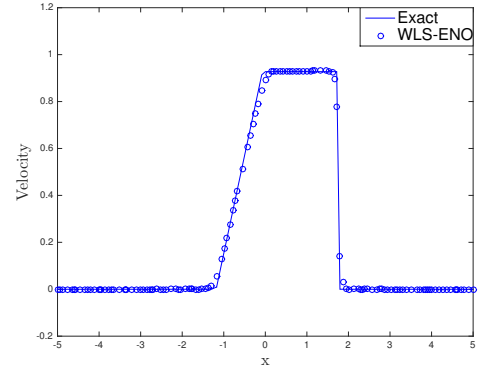

(c) Velocity.

Figure 8: Solutions of 1-D Euler equations at $t=1$ with fifth-order WLS-ENO on non-uniform grid.

Interacting Blast Waves. Next, we consider the 1-D blast wave problem [37], which has the initial condition

$$
(\rho, u, P)= \begin{cases}(1,0,1000) & 0 \leq x<0.1 \\ (1,0,0.01) & 0.1 \leq x<0.9 \\ (1,0,100) & 0.9 \leq x<1\end{cases}
$$

and reflective boundary conditions at both sides. For this test, sharp resolution of discontinuities is critical for the accuracy of the overall flow solution. Figure 9 shows the result using fifth-order WLS-ENO scheme at $t=0.038$, which agreed with the exact solution very well.

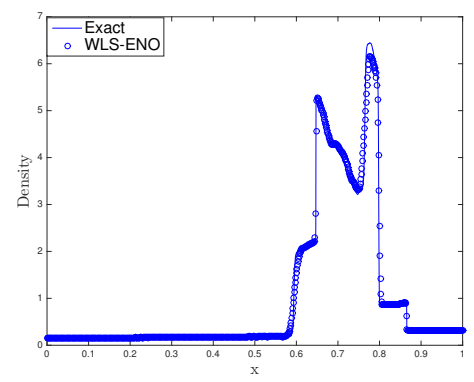

(a) Density.

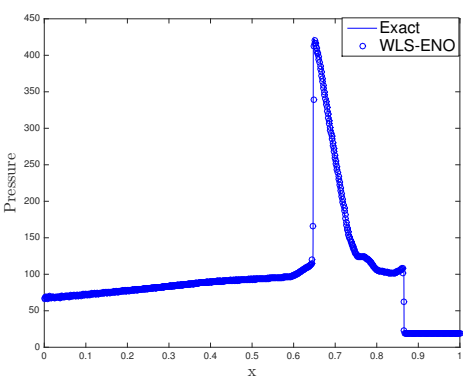

(b) Pressure.

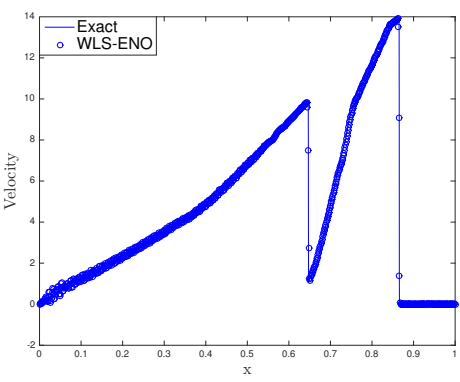

(c) Velocity.

Figure 9: Solutions of 1-D interacting blast waves at $t=0.038$ with fifth-order WLS-ENO.

\subsection{2-D Results}

We now present results of WLS-ENO with 2-D unstructured meshes for problems with smooth or piecewise smooth solutions, including the wave equation, Burgers' equation, and the Euler equations with two different initial conditions.

\subsubsection{2-D Wave Equation}

As in 1-D, we first consider the wave equation,

$$
u_{t}+u_{x}+u_{y}=0,
$$


with periodic boundary conditions and the initial condition

$$
u_{0}(x, y)=\sin \left(\frac{\pi}{2}(x+y)\right), \quad-2 \leq x \leq 2,-2 \leq y \leq 2 .
$$

For this problem, the solution remains smooth over time. We solve the problem using third-order WLS-ENO scheme and third-order WENO scheme. Figure 11 shows the errors at $t=1$, and it can be seen that both methods achieved only second order convergence. This convergence rate is expected, because the derivatives can only be approximated to second-order accurate by polynomial approximations over nonuniform unstructured meshes without symmetry. When applying WLS-ENO on a uniform mesh, such as that shown in Figure 12, it would deliver the convergence rate one order higher due to error cancellation, similar to WENO and other finite difference methods, as illustrated with the fourth-order WLS-ENO scheme and fourth-order WENO scheme in Figure 13. Note that on uniform meshes, WLS-ENO may be slightly less accurate than WENO because it uses a larger stencil.

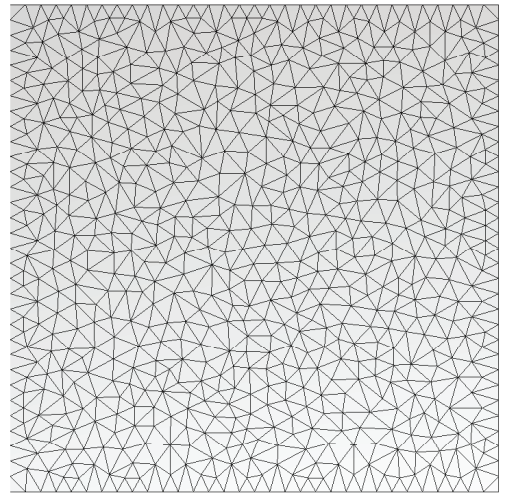

Figure 10: Sample unstructured triangular mesh for solving 2-D wave equation.

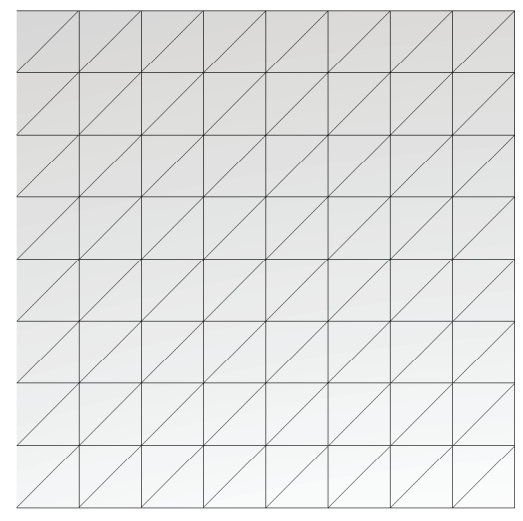

Figure 12: Sample uniform triangular mesh for solving 2-D wave equation.

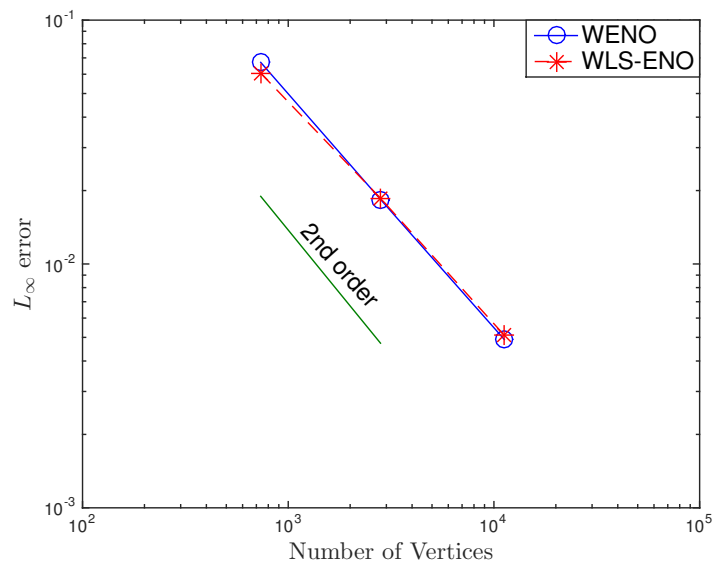

Figure 11: Errors in numerical solutions of 2-D wave equation with WENO and WLS-ENO at $t=1$.

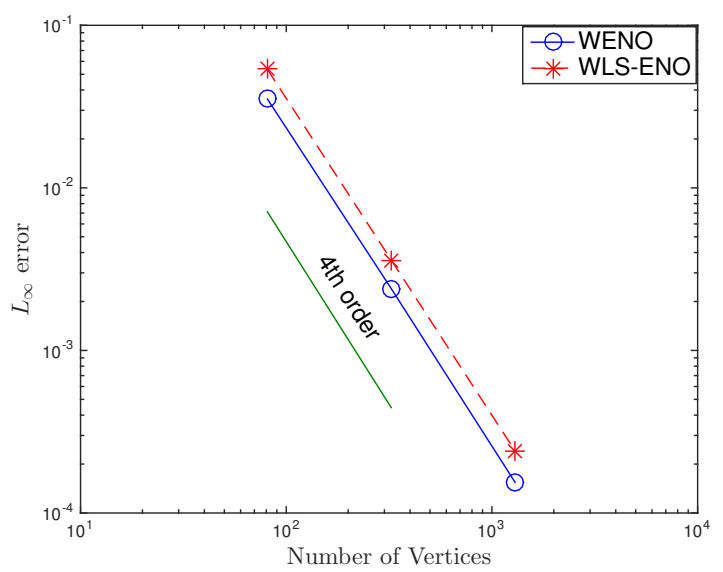

Figure 13: Errors in numerical solutions of $2-\mathrm{D}$ wave equation with WENO and WLS-ENO on uniform meshes. 


\subsubsection{2-D Burgers' Equation}

For piecewise smooth solutions, we solve the 2-D Burgers' equation

$$
u_{t}+\left(\frac{u^{2}}{2}\right)_{x}+\left(\frac{u^{2}}{2}\right)_{y}=0
$$

over $[-2,2]^{2}$, with periodic boundary conditions and the initial condition

$$
u_{0}(x, y)=0.3+0.7 \sin \left(\frac{\pi}{2}(x+y)\right) .
$$

Although the initial condition is smooth, discontinuities develop over time. Figure 14(left) shows the exact solution at $t=0.5$, when the solution becomes discontinuous, and Figure 14(right) shows the result of fourth-order WLS-ENO scheme under non-uniform grid refinement. It can be seen that the overall solution remained accurate as discontinuities developed.
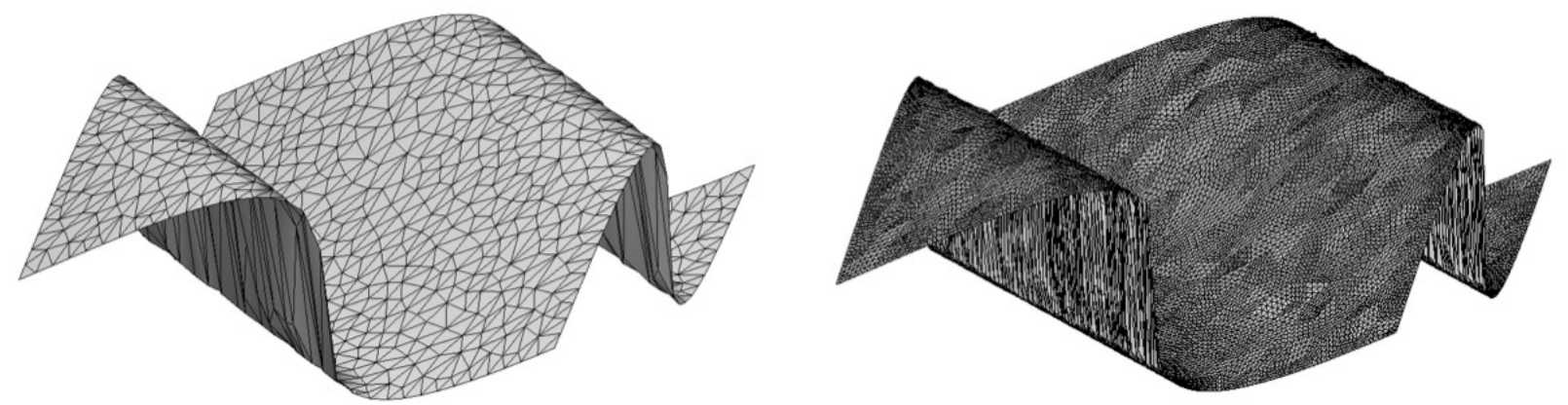

Figure 14: Exact (left) and numerical solutions (right) with fourth-order WLS-ENO for 2-D Burgers' equation at $t=0.5$.

\subsubsection{2-D Euler Equations}

The 2-D Euler equations have the following form

$$
\left(\begin{array}{c}
\rho \\
\rho u \\
\rho v \\
E
\end{array}\right)_{t}+\left(\begin{array}{c}
\rho u \\
\rho u^{2}+p \\
\rho u v \\
u(E+p)
\end{array}\right)_{x}+\left(\begin{array}{c}
\rho v \\
\rho u v \\
\rho v^{2}+p \\
v(E+p)
\end{array}\right)_{y}=\mathbf{0}
$$

where

$$
E=\frac{p}{\gamma-1}+\frac{1}{2} \rho\left(u^{2}+v^{2}\right) .
$$

In our tests, we use $\gamma=1.4$ as in 1-D, and use characteristic decomposition to split variables as described in [5]. WLS-ENO is then applied to each of the characteristic field.

Vortex Evolution Problem. This is one of the few problems that has exact solutions for the compressible Euler equations. The test case involves the convection of an isentropic vortex in inviscid flow, and it tests the ability of numerical schemes to capture vortical flows. We consider an idealized setting over $[0,10]^{2}$ with periodic boundary conditions. The mean flow is $\rho_{\infty}=1, p_{\infty}=1$, and $\left(u_{\infty}, v_{\infty}\right)=(1,1)$. For the initial condition, we place an isentropic vortex to the mean flow field. The perturbation values are given by

$$
\begin{gathered}
(\delta u, \delta v)=\frac{\beta}{2 \pi} e^{\frac{1-r^{2}}{2}}(-\bar{y}, \bar{x}), \\
\delta T=-\frac{(\gamma-1) \beta^{2}}{8 \gamma \pi^{2}} e^{1-r^{2}}
\end{gathered}
$$

where $(\bar{x}, \bar{y})=(x-5, y-5), r^{2}=\bar{x}^{2}+\bar{y}^{2}$, and the vortex strength $\beta=5$. The exact solution of this problem convecting the vortex along the diagonal direction. We used fifth-order WLS-ENO scheme and computed the results up to $t=1$. Figure 15 shows the result, which achieved the expected convergence rate. 


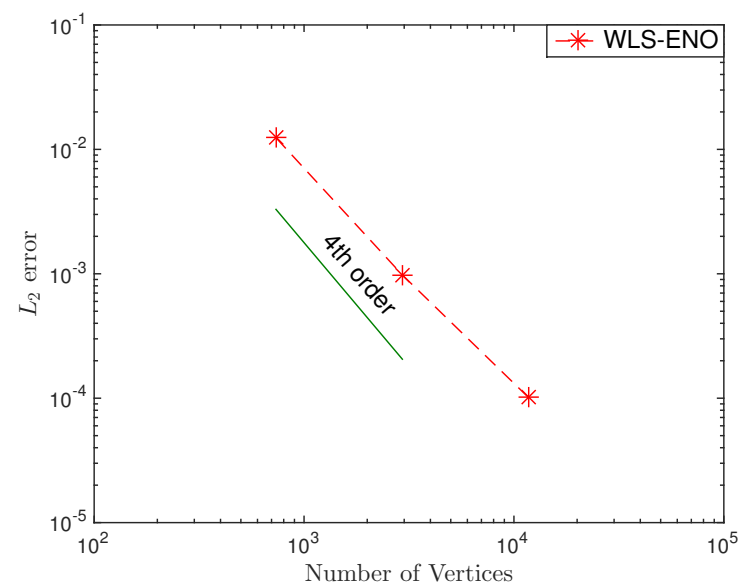

Figure 15: Convergence result of $\rho$ for 2-D vortex evolution problem with fifth order WLS-ENO on triangular meshes.

Explosion Test Problem. In this test, we solve the 2-D explosion test [38, 9], which solves the Euler equations over a unit disk centered at the origin. The initial condition is given by

$$
(\rho, u, v, p)^{T}=\left\{\begin{array}{ll}
(1,0,0,1)^{T} & \sqrt{x^{2}+y^{2}} \leq 0.2 \\
(0.125,0,0.1)^{T} & \sqrt{x^{2}+y^{2}}>0.2
\end{array} .\right.
$$

We triangulated the unit disk with meshes similar to that in Figure 10, and ran the test up to $t=0.1$ to ensure that the explosion waves do not reach the boundary. To obtain a reference solution, note that this problem is mathematically equivalent to the axisymmetric Euler equations [39]

$$
\frac{\partial}{\partial t}\left(\begin{array}{c}
\rho \\
\rho u \\
E
\end{array}\right)+\frac{\partial}{\partial r}\left(\begin{array}{c}
\rho u \\
\rho u^{2}+p \\
u(E+p)
\end{array}\right)=-\frac{d-1}{r}\left(\begin{array}{c}
\rho u \\
\rho u^{2} \\
u(E+p)
\end{array}\right)
$$

where $r$ is the radial coordinate. We solved this 1-D problem on a very fine mesh composed of 4,000 grid points and use its solution as the reference. Figures 16 and 17 show the numerical solution for the density at $t=0.1$ with the third-order WLS-ENO scheme. The results agreed very well with the 1 -D solutions.

\subsection{3-D Results}

One advantage of WLS-ENO is that it generalizes to 3-D in a straightforward fashion. We present some numerical results over unstructured meshes in 3-D, including the wave equation, Burgers' equation, and the Euler equations.

\subsubsection{3-D Wave Equation}

We first solve the 3 -D linear wave equation

$$
u_{t}+u_{x}+u_{y}+u_{z}=0
$$

over $[-2,2]^{3}$, with periodic boundary conditions and the initial condition

$$
u(x, y, z, 0)=\sin \left(\frac{\pi}{2}(x+y+z)\right) .
$$

We solve the problem using WLS-ENO over a series of unstructured meshes, where the coarsest mesh is depicted in Figure 18. Figure 19 shows the errors with third-order and fourth-order WLS-ENO schemes under mesh refinement. It is clear that both schemes achieved the convergence rate close to three, and the error of fourth-order WLS-ENO scheme was about half of that of the third-order scheme. 


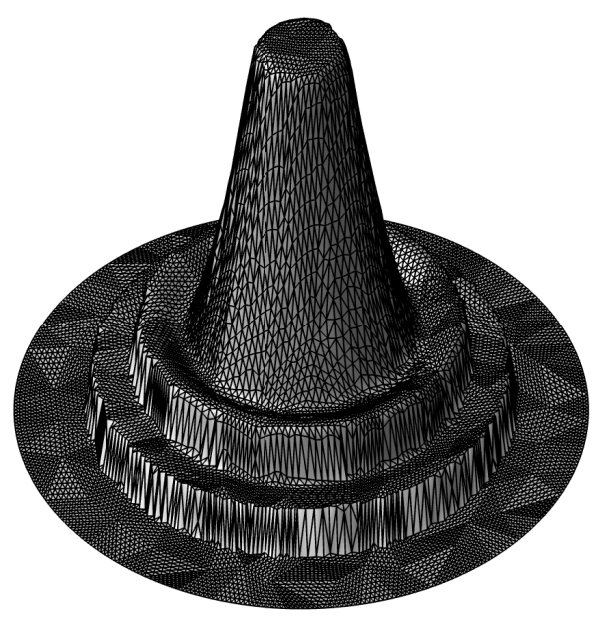

Figure 16: Numerical solution of $\rho$ for 2-D explosion test with third-order WLS-ENO at $t=0.1$.

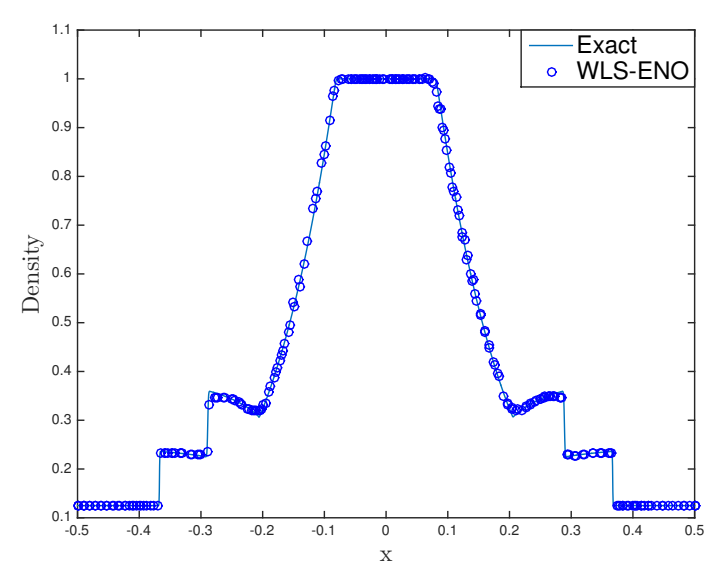

Figure 17: Numerical solution of $\rho$ along $x$ axis of 2-D explosion test with third-order WLS-ENO vs. 1-D solution at $t=0.1$.

\subsubsection{3-D Burgers' Equation}

In this test, we solve the 3-D nonlinear Burgers' equation

$$
u_{t}+\left(\frac{u^{2}}{2}\right)_{x}+\left(\frac{u^{2}}{2}\right)_{y}+\left(\frac{u^{2}}{2}\right)_{z}=0
$$

over $[-2,2]^{3}$, also with periodic boundary conditions and the initial condition

$$
u(x, y, z, 0)=0.3+0.7 \sin \left(\frac{\pi}{2}(x+y+z)\right) .
$$

Similar to the 2-D case, discontinuities develop at $t=0.5$. Figure 20 shows a $1-\mathrm{D}$ cross section of the numerical solutions along $x=y$ and $z=0$ with third-order and fourth-order WLS-ENO schemes at $t=0.5$, overlaid with the exact solution. Both solutions are non-oscillatory. In contrast, the third-order WENO scheme in [30] was unstable over a non-uniform unstructured mesh. On a tetrahedral mesh obtained by decomposing a structured mesh, WLS-ENO and WLS-ENO achieved comparable accuracy for the problem.

\subsubsection{3-D Euler Equations}

As our final test, we solve the 3 -D version of the explosion test $[38,9]$. The 3-D Euler equations have the form

$$
U_{t}+\frac{\partial F}{\partial x}+\frac{\partial G}{\partial y}+\frac{\partial H}{\partial z}=0
$$

where

$$
U=\left(\begin{array}{c}
\rho \\
\rho u \\
\rho v \\
\rho w \\
E
\end{array}\right), \quad F=\left(\begin{array}{c}
\rho u \\
\rho u^{2}+p \\
\rho u v \\
\rho u w \\
u(E+p)
\end{array}\right), \quad G=\left(\begin{array}{c}
\rho v \\
\rho u v \\
\rho v^{2}+p \\
\rho v w \\
v(E+p)
\end{array}\right), \quad H=\left(\begin{array}{c}
\rho w \\
\rho u w \\
\rho v w \\
\rho w^{2}+p \\
w(E+p)
\end{array}\right)
$$

and

$$
E=\frac{p}{\gamma-1}+\frac{1}{2} \rho\left(u^{2}+v^{2}+w^{2}\right) .
$$




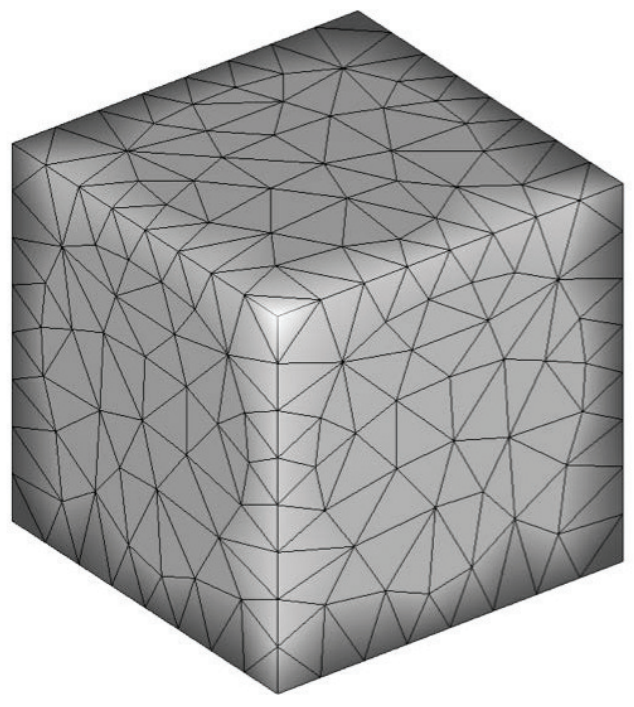

Figure 18: Sample unstructured mesh for solving 3-D wave equations.

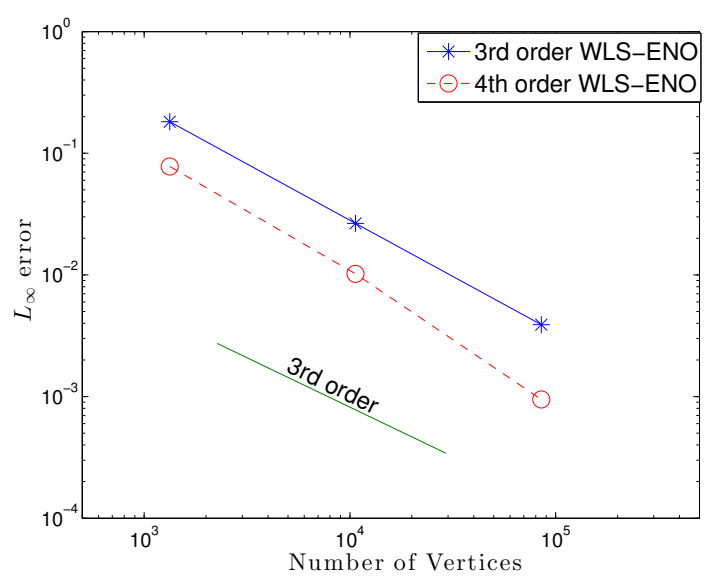

Figure 19: Convergence of third- and fourth-order WLS-ENO schemes for wave equation on tetrahedral meshes.

As in 1-D and 2-D, we chose $\gamma=1.4$. The computational domain is a unit ball centered at the origin, which we tessellate with a tetrahedral mesh. The initial condition of this problem is given by

$$
(\rho, u, v, w, p)^{T}=\left\{\begin{array}{ll}
(1,0,0,0,1)^{T} & \sqrt{x^{2}+y^{2}+z^{2}} \leq 0.2 \\
(0.125,0,0,0,0.1)^{T} & \sqrt{x^{2}+y^{2}+z^{2}}>0.2
\end{array} .\right.
$$

We solved the problem in a component-by-component fashion up to $t=0.1$, and obtained a reference solution by solving the 1-D problem (92). Figures 22 and 23 show the numerical solutions of the density at $t=0.1$, which agreed with the 1-D solutions very well.

\section{Conclusions and Future Work}

In this paper, we introduced a new family of essentially non-oscillatory schemes, called WLS-ENO, in the context of finite volume methods for solving hyperbolic conservation laws. The schemes are derived based on Taylor series expansions and solved with a weighted least squares formulation. They can be applied to both structured and unstructured meshes. Over structured meshes, we showed that WLS-ENO delivers similar and even better accuracy compared to WENO, while enabling a larger stability region. For unstructured meshes, we showed that WLS-ENO enables accurate and stable solutions. Its accuracy and stability are rooted in the facts that the convexity requirement is satisfied automatically in WLS-ENO, and the stencil can be adapted more easily to ensure the stability of the approximations. We presented detailed analysis of WLS-ENO in terms of accuracy in 2-D, and its stability for hyperbolic conservation laws in 1-D. We also assessed the WLS-ENO with a large collection of test problems in 1-D, 2-D, and 3-D, including wave equations, Burgers' equation, and the Euler equations with fairly complicated initial conditions. Our numerical results demonstrated that WLS-ENO is accurate and stable over unstructured meshes for very complex problems.

In its current form, WLS-ENO still has some limitations. Its primary disadvantage is that it has higher computational cost compared to the traditional WENO scheme over structured meshes. However, for engineering applications involving complex geometries, WLS-ENO provides a more general tool for dealing with piecewise smooth functions over unstructured meshes. To improve efficiency, it is also possible to develop a hybrid method that utilizes the traditional WENO on structured meshes in the interior and utilizes WLSENO over unstructured meshes near complex boundaries. Another limitation of the present formulation of 


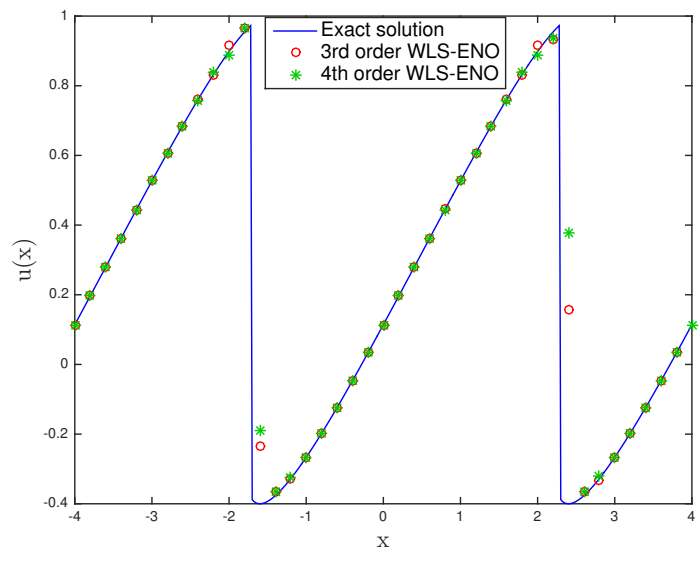

Figure 20: 1-D cross-sections of 3-D Burgers' equation using third- and fourth-order WLS-ENO schemes at $t=0.5$.

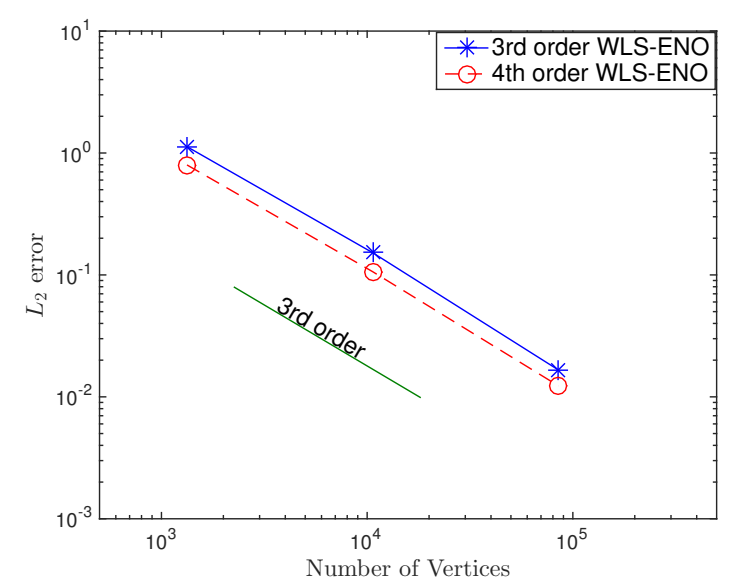

Figure 21: Convergence of third- and fourth-order WLS-ENO for Burgers' equation at $t=0.5$ away from singularities.

WLS-ENO is that it only applies to finite volume methods. As future work, we will extend WLS-ENO to support generalized finite difference methods on unstructured meshes, optimize the performance of WLS-ENO, and apply the methods to applications in computational fluid dynamics.

\section{Acknowledgements}

This work was supported by DoD-ARO under contract \#W911NF0910306. The second author is also supported by a subcontract to Stony Brook University from Argonne National Laboratory under Contract DE-AC02-06CH11357 for the SciDAC program funded by the Office of Science, Advanced Scientific Computing Research of the U.S. Department of Energy.

\section{References}

[1] S. Gottlieb, C.-W. Shu, Total variation diminishing Runge-Kutta schemes, Math. Comput. 67 (221) (1998) 73-85.

[2] C.-W. Shu, Essentially non-oscillatory and weighted essentially non-oscillatory schemes for hyperbolic conservation laws, in: E. A. Quarteroni (Ed.), Advanced Numerical Approximation of Nonlinear Hyperbolic Equations, Vol. 1697 of Lecture Notes in Mathematics, Springer, 1998, pp. 325-432.

[3] G. Jiang, C.-W. Shu, Efficient implementation of weighted ENO schemes, J. Comput. Phys. 126 (1996) $202-228$.

[4] C.-W. Shu, High order weighted essentially nonoscillatory schemes for convection dominated problems, SIAM Rev. 51 (1) (2009) 82-126.

[5] C. Hu, C.-W. Shu, Weighted essentially non-oscillatory schemes on triangular meshes, J. Comput. Phys. 150 (1999) 97-127.

[6] D. Levy, G. Puppo, G. Russo, A fourth-order central WENO scheme for multidimensional hyperbolic systems of conservation laws, SIAM J. Sci. Comput. 24 (2) (2002) 480-506.

[7] J. Shi, C. Hu, C.-W. Shu, A technique of treating negative weights in WENO schemes, J. Comput. Phys. 175 (2002) 108-127. 


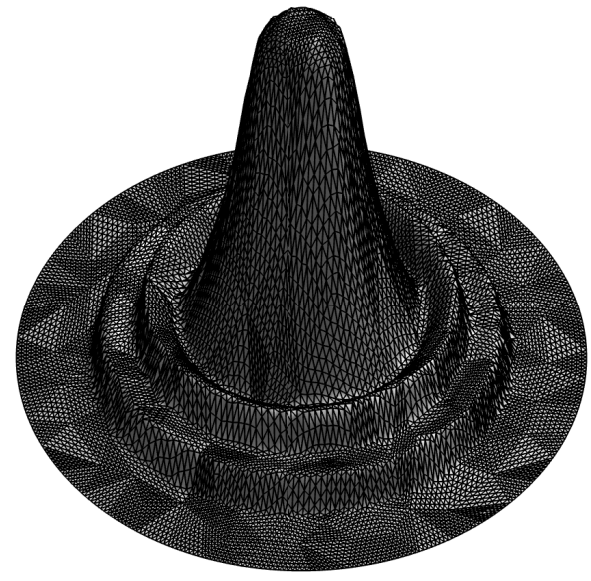

Figure 22: Cross section of numerical solution of $\rho$ in $x y$ plane of 3-D explosion test with third-order WLS-ENO at $t=0.1$.

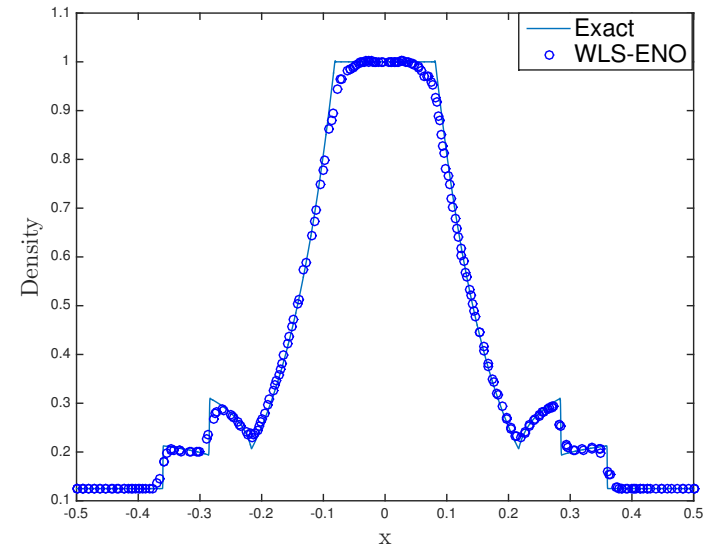

Figure 23: Numerical solution of $\rho$ along $x$ axis of 3-D explosion test with third-order WLS-ENO vs. 1-D solution at $t=0.1$.

[8] P. Tsoutsanis, V. A. Titarev, D. Drikakis, WENO schemes on arbitrary mixed-element unstructured meshes in three space dimensions, J. Comput. Phys. 230 (4) (2011) 1585-1601.

[9] M. Lahooti, A. Pishevar, A new fourth order central WENO method for 3D hyperbolic conservation laws, Appl. Math. Comput. 218 (20) (2012) 10258-10270.

[10] V. Titarev, E. Toro, Finite-volume WENO schemes for three-dimensional conservation laws, J. Comput. Phys. 201 (1) (2004) 238-260.

[11] A. K. Henrick, T. D. Aslam, J. M. Powers, Mapped weighted essentially non-oscillatory schemes: Achieving optimal order near critical points, J. Comput. Phys. 207 (2005) 542-567.

[12] R. Borges, B. Costa, W. S. Don, An improved weighted essentially non-oscillatory scheme for hyperbolic conservation laws, J. Comput. Phys. 227 (2008) 3191-3211.

[13] S. Zhang, C.-W. Shu, A new smoothness indicator for the WENO schemes and its effect on the convergence to steady state solutions, J. Comput. Phys. 31 (1-2) (2007) 273-305.

[14] Y. Shen, G. Zha, Improvement of the WENO scheme smoothness estimator, Int. J. Numer. Meth. Fluid 64 (6) (2010) 653-675.

[15] F. Grasso, S. Pirozzoli, Shock-wave vortex interactions: Shock and vortex deformations, and sound production, Theoret. Comput. Fluid Dynamics 13 (2000) 421-456.

[16] J.-Y. Yang, S.-C. Yang, Y.-N. Chen, C.-A. Hsu, Implicit weighted ENO schemes for the threedimensional incompressible Navier-Stokes equations, J. Comput. Phys. 146 (1) (1998) 464-487.

[17] G.-S. Jiang, D. Peng, Weighted ENO schemes for Hamilton-Jacobi equations, SIAM J. Sci. Comput. 21 (6) (2000) 2126-2143.

[18] R. Liska, B. Wendroff, Two dimensional shallow water equations by composite schemes, Int. J. Numer. Meth. Fluids 30 (1999) 461-479.

[19] Y. Liu, Y.-T. Zhang, A robust reconstruction for unstructured WENO schemes, J. Sci. Comput. $54(2,3)$ (2013) 603-621.

[20] A. J. Christlieb, Y. Liu, Q. Tang, Z. Xu, High order parametrized maximum-principle-preserving and positivity-preserving WENO schemes on unstructured meshes, J. Comput. Phys. 281 (2015) 334-351. 
[21] M. R. Charest, T. R. Canfield, N. R. Morgan, J. Waltz, J. G. Wohlbier, A high-order vertex-based central ENO finite-volume scheme for three-dimensional compressible flows, Comput. Fluids 114 (2015) $172-192$.

[22] J. Zhu, J. Qiu, Finite volume Hermite WENO schemes for solving the Hamilton-Jacobi equations II: Unstructured meshes, Comput. Math. Appl. 68.10 (2014) 1137-1150.

[23] G. Sod, Numerical Methods in Fluid Dynamics, Cambridge University Press, Cambridge, 1985.

[24] R. LeVeque, Numerical Methods for Conservation Laws, Birkhäuser Verlag, Basel-Boston-Berlin, 1992.

[25] A. Harten, S. Osher, B. Engquist, S. Chakravarthy, Uniformly high order accurate essentially nonoscillatory schemes, III, J. Comput. Phys. 71 (1987) 231-303.

[26] M. Castro, B. Costa, W. S. Don, High order weighted essentially non-oscillatory WENO-Z schemes for hyperbolic conservation laws, J. Comput. Phys. 230 (2011) 1766-1792.

[27] S. Zhang, C.-W. Shu, A new smoothness indicator for the WENO schemes and its effect on the convergence to steady state solutions, SIAM J. Sci. Comput. 31 (1-2) (2007) 273-305.

[28] Y. Shen, G. Zha, Improvement of the WENO scheme smoothness estimator, Int. J. Numer. Meth. Fluid 64 (6) (2010) 653-675.

[29] W. Wolf, J. Azevedo, High-order ENO and WENO schemes for unstructured grids, Int. J. Numer. Meth. Fluid 55 (10) (2007) 917-943.

[30] Y.-T. Zhang, C.-W. Shu, Third order WENO scheme on three dimensional tetrahedral meshes, Commun. Comput. Phys. 5 (2-4) (2009) 836-848.

[31] M. R. Charest, C. P. Groth, P. Q. Gauthier, High-order CENO finite-volume scheme for low-speed viscous flows on three-dimensional unstructured mesh, Convergence 59 (2012) 60.

[32] X. Jiao, D. Wang, Reconstructing High-Order Surfaces for Meshing, in: S. Shontz (Ed.), Proceedings of the 19th International Meshing Roundtable, Springer Berlin Heidelberg, 2010, pp. 143-160.

[33] R. Conley, T. J. Delaney, X. Jiao, Overcoming element quality dependence of finite elements with adaptive extended stencil FEM (AES-FEM), Int. J. Numer. Meth. Engrg. To appear. Preprint available at http://arxiv.org/abs/1508.01435.

[34] J. S. Park, S.-H. Yoon, C. Kim., Multi-dimensional limiting process for hyperbolic conservation laws on unstructured grids, J. Comput. Phys. 229 (2010) 788-812.

[35] V. Dyedov, N. Ray, D. Einstein, X. Jiao, T. J. Tautges, AHF: Array-based half-facet data structure for mixed-dimensional and non-manifold meshes, in: J. Sarrate, M. Staten (Eds.), Proceedings of the 22nd International Meshing Roundtable, Springer International Publishing, 2014, pp. 445-464.

[36] R. J. LeVeque, Nonlinear conservation laws and finite volume methods, in: O. Steiner, A. Gautschy (Eds.), Computational Methods for Astrophysical Fluid Flow, Vol. 27 of Saas-Fee Advanced Courses, Springer, 1998, pp. 1-159.

[37] P. Woodward, P. Colella, Numerical simulation of two-dimensional fluid flows with strong shocks, J. Comput. Phys. 54 (1984) 115.

[38] M. Dumbser, O. Zanotti, A. Hidalgo, D. S. Balsara, ADER-WENO finite volume schemes with spacetime adaptive mesh refinement, J. Comput. Phys. 248 (2013) 257-286.

[39] E. F. Toro, Riemann Solvers and Numerical Methods for Fluid Dynamics: A Practical Introduction, Springer, 2009. 